$Q L$
555
$G 7 D G$
1862

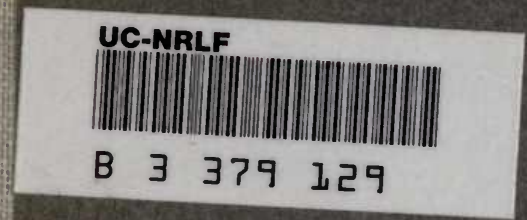




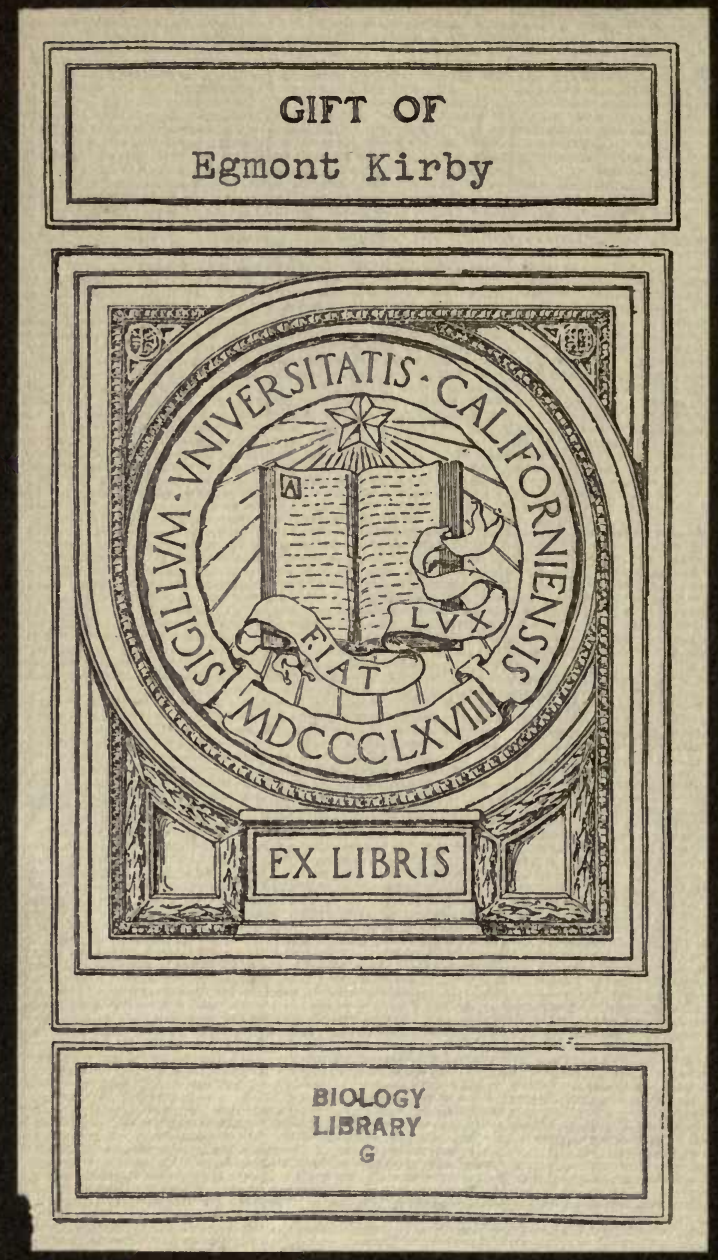




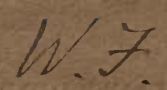

A SYNONYMIO LIST

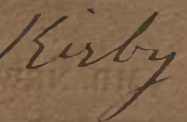

8

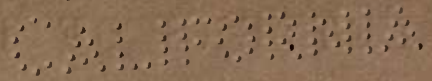

OF ALL TIYE

$$
\text { UAN: } \operatorname{ILT}_{1925}
$$

\title{
IITISH BUTTERFLIES
}

\author{
AND \\ MOTHS.
}

BY HENRY DOUBLEDAY.

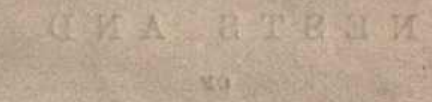

SECOND EDITION.

LONDON :

WARD NEWMAN, 9, DEVONSHIRE STREET, BISHOPSGATE.

Price SIXPENCE.

The same work is published in duplicate, that is two copies bound in one. One copy is precisely the same as this; the other is printed on one side only, and on strong, stout paper, intended for cutting out and labelling cabinets. Price TWO SHILLINGS. 
MR. NEW.NA T'S WORKS ON BRITISH NATURAL HISTORY. $\because \because: \because \vdots \vdots \vdots \vdots: 0$

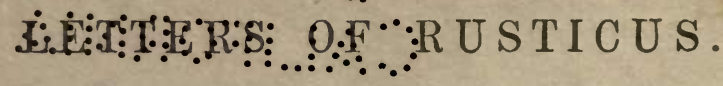

Hollow, sandy Lanes; Birds' Nests; Wild Cat; Black Grouse; Sand Martin ; Hooded Crow; Ring Ouzel; Migration of Birds; Screech Owl; Woodcock ; Water Rail ; Eared Grebe; Moorhen; Titmouse ; Dabchick; Great Northern Diver ; Wild Swan; Singing of Swans; Fern Owl; Furze Wren; Crossbill; Siskin, Lesser Redpole ; Chaffinch, Blackcap ; Nightingale; Trip to the Isle of Wight; Freshwater Gate ; Fresh-water Cliffs ; Multitude of Birds; Colony of Cormorants ; Passage through the Needles; Lobster Pots; Sea-birds' Eggs; Egg Collectors; Eggs stolen by Gulls, and how; Eggs stolen by Ravens, and how; Crab Race; Ring Dutterels and Purres; Migration of Puffin; Insects injurious to Vegetables; Gooseberry Grub, The Fly described, Laying the Eggs; The young Grub; Voracity of the Grubs; the Grub described; Change of Skin; Burrows in the Earth; Remedies; Aphis or Plant-Louse ; Injury it causes; Economy of; Hop-Fly; Influence on Crop of Hops; Influence on the Duty; First Appearance of; Insect Euemies of ; Remedies; American Blight; Apple-Weevil; Description of; Economy of ; Remedies; Apple-Moth; Apple-Grub: Economy of; Little Ermine Moth; Yellow-Tail ; Turnip-Fly ; Destructive Powers of ; Economy of ; Remedies; Turnip-Nigger ; Description of Parent Fly ; Economy of ; Description of the Grub; Remedies; Turnip-Weevil; Turnip-Aphis; Aphides in Apples, in Poplar-leaves and on Roots ; Ants and Aphides; Hedgehog; Hybernation of ; Error in defending it as an Herbivorous Animal; Destroys Vipers, and is generally Carnivorous; Longtailed Tit; Dabchick; Hare; Weasel hunts Rats, as well as Rabbits ; Flowering of the Aspen; Coccus of the Vine; Flies and Humble Bees; Burying Beetle.

Price 8 s. $6 \mathrm{~d}$., post free.

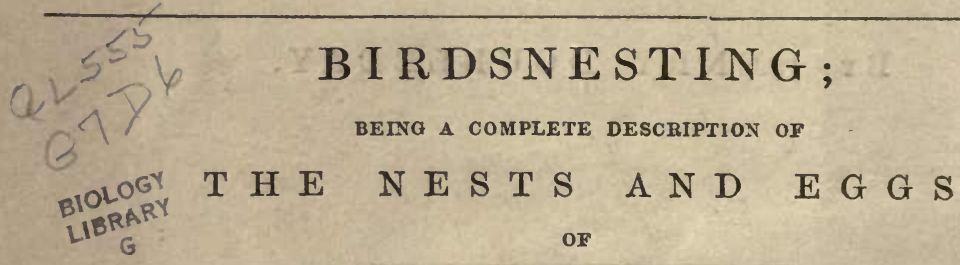

\section{BIRDS WHICH BREED IN GREAT BRITAIN AND IRELAND.}

BY EDWARD NEWMAN, F.L.S., F.Z.S.

This work is written expressly for the use of out-of-doors naturalists and that better class of schoolboys who take up Natural History as an instructive recreation. Under each species will be found

1. The English name.

2. The Latin name.

3. The situation in which the nest is to be found.

4. The materials of which the nest is built, together with any particulars of its shape which will assist in determining by what bird it is built.

5. The number and colour of the eggs, describing such variations as are occasion. ally met with.

Price 1s. 1d., post free. 


\section{SYNONYMIC LIST}

OF

\section{BRITISH LEPIDOPTERA:}

\section{DIURNI.}

PAPILIONIDAE, Gn. PAPILIO, Linn.

Machann.

Machaon, Linn.

\section{PIERIDAE, Gn.}

LEUCOPHASIA, Steph.

Sinapis.

Sinapis, Linn.

\section{PIERIS, Schr.}

\section{Crategr.}

Cratægi, Linn.

Brassic

Brassicæ, Linn.

Chariclea, Steph. (var, ver.)

RAPE.

Rapæ, Linn.

Metra, Steph. (var. ver.)

NAPI.

Napi, Linn.

Napææ, Steph., var.

Sabellicæ, Steph., var.

Daplidice.

Daplidice, Linn.

ANTHOCHARIS, $B d v$.

Cardamines.

Cardamines, Lınn.

RHODOCERIDE, Gn. GONEPTERYX, Leach.

RhaMNi.

Rhamni, Linn.
COLIAS, $B d v$.

Enusa.

Edusa, Fab.

Chrysotheme, Steph., var.

Tar. \&. Helice, Hub.

HYALE.

Hyale, Linn.

VANESSID AE, Gn. ARGYNNIS, Och.

Paphia.

Paphia, Linn.

Var. \&. Valezina, Esp.

AgraJA.

Aglaja, Linn.

Charlotta, Sowerby, var.

Adippe.

Adippe, $F a b$.

IATHONIA.

Lathonia, Linn.

Euphiosyne.

Euphrosyne, Linn.

Selene.

Selene, $F a b$.

MELITEA, Fab.

Artemis.

Artemis, Fab.

\section{Cinxia.}

Cinxia, Linn.

Athalia.

Athalia, Esp.

Dietynna, Haw.

Eos, Haw., var.
VANESSA, Och.

C-album.

C-album, Linn.

URTIOE.

Urticæ, Linn.

Polychloros.

Polychloros, Linn.

Antiopa.

Antiopa, Linn.

Io.

Io, Linn.

Atalanta.

Atalanta, Linn.

Cardur.

Cardui, Linn.

NYMPHALIDE, Gn. LIMENITIS, $B d v$.

SibYLLA.

Sibylla, Linn.

Camilla, Haw.

APATURA, $O c h$.

IrIs.

Iris, Linn.

SATYRIDE, G॥.

ARGE, Esp.

Galathea.

Galathes, Linn.

\section{EREBIA, Bdv.}

Casstope.

Cassiope, Fab.

Mnemon, Hawo.

Melampus, Newm., var. 


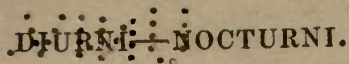

\section{Blaxdina. Blandina, Fab.}

SATYRUS, $B d v$.

Egeria

Ægeria, Linn.

Megera.

Megæra, Linn.

SEMELE.

Semele, Linn.

JAviRA.

Janira, Linn.

\& Jurtina, Linn.

Tithones.

Titbonus, Linn.

HyPERANTHUS.

Hyperanthus, Linn.

\section{Chortobius, $\boldsymbol{G} n$.}

Davus.

Davus, Fab.

Polydama, Haw , var.

Typhon, Haw., var.

Paiphilus.

Pamphilus, Linn.

\section{$L Y C A E N I D A, G n$.} THECLA, Fab.

\section{Ruвi.}

Rubi, Linn.

Quercus.

Quercus, $\operatorname{Linn}$.

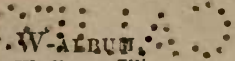

W-album, Illig.

Pruni, Haw.

Pruni.

Pruni, Linn.

Betula.

Betulæ, Linn.

POLYOMMATUS, $B d v$.

Hippothoе.

Hippothoe, Linn.

Dispar, Haw.

Phleas.

Pblæas, Linn.

LYCENA, $T r$.

\section{EGON.}

Ægon, Bork.

Argus, Haw.

Agestis.

Agestis, W. $V$.

Idas, Haw.

Artaxerxes, Fab., var.

Allous, $H u b$., var.

Salmacis, Steph., var.

Alexis.

Alexis, $H u b$.

Eros, Steph., var.

Icarius, Steph., var.

ADonis.

Adonis, Fab.

Hyacinthus, Lewin, var.

Corydon.

Corydon, Fab.

Acis.

Acis, $W . V$.

Cymon, Haw.
Ar.sus

Alsus, Fab.

Argiolus.

Argiolus, Linn.

ArIon.

Arion, Linn.

ER YCINID EE, Gn. NEMEOBIUS, Steph.

I.UCINA.

Lucina, Linn.

HESPERID E, Gn. SYRICTHUS, $B d v$.

Alveolus.

Alveolus, Hub.

Malvæ, Haw.

Fritillum, Lewin, var.

THANAOS, $B d v$.

TAGes.

Tages, Linn.

HESPERIA, $B d v$.

Paniscus.

Paniscus, Fab."

Sylvanus.

Sylvanus, Fab.

Com ma.

Comma, Linn.

LiNEA.

Linea, Fab.

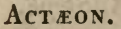

Actæon, Esp.

\section{NOCTURNI, Gn.}

\section{SPHINGES AND BOMBYCES OF LINNEUS.}

\section{SPHINGIDAE, Gn.} S.MERINTHUS, Lat.

\section{Oceltatus.}

Ocellata, Linn.

Salicis, Hub.

Populi.

Populi, Linn.

TILIF.

Tiliæ, Linn.

ACHERONTIA, Och.

Atropos.

Atropos, Linn.

SPHINX, Linn.

Coxvolvur.I.

Cunvolvuli, Linn.
Ligustri.

Ligustri, $\operatorname{Lin} n$.

DEILEPHILA, Och.

EUPHORBIE.

Euphorbiæ, Linn.

GALII.

Galii, W. V.

LINEATA.

Lineata, $F u b$.

Livornica, Esp.

CHEROCAMPA, Dup.

Celerio.

Celerio, Linn.

Porcellus.

Porcellus, Linn.
ELPENor.

Elpenor, Linn.

NerIt.

Nerii, Linn.

MACROGLOSSA, Och.

Stellatarum.

Stellatarum, Linn.

Fuciformis.

Fuciformis, $\operatorname{Linn}$.

Bombyliformis, Och.

BOMBYLIFORMIS.

Bombyliformis, $E s p$. Fuciformis, Steph. 


\section{SESIDAE, Gn.}

SESIA, Fab.

MYopaformis.

Myopæformis, Bork.

Mutillæformis, Lasp.

Culiciformis.

Culiciformis, Linn.

Formic AfFormis.

Formicæformis, Esp.

Chirysidiformis.

Chrysidiformis, Esp.

ICHNEUMONIFORMIS.

Ichoeumoniformis, $W . V$.

Cynipiformis.

Cynipiformis, Och.

Chry sorrhæa, Don.

TPulfForMis.

Tipuliformis, Linn.

Allantiformis.

Allantiformis, Newn.

Andreniformis, Lasp.?

Scolieformis.

Scoliæformis, $H u b$.

Spheciformis.

Spheciformis, $W . V$.

Asiliformis.

Asiliformis, $W . V$.

Estriformis, Kirby.

Vespiformis, Linn.?

BeMBECIFormis.

Bembeciformis, $\boldsymbol{H} u \boldsymbol{b}$.

Crabroniformis, Lewin.

A PIFormis.

Apiformis, Linn.

ZEUZERIDA, $\mathrm{Gn}$. MACROGASTER, Dup.

Arundinis.

Arundinis, $H u b$.

ZEUZERA, Lat.

AEscULI.

Ėsculi, Linn.

COSSUS, Fab.

LIG NIPERDA.

Ligniperda, $F a b$.

HEPIALIDAE, Gn. HEPIALUS, Fab.

Hectus.

Hecta, Linn.

\& Jodutta, Haw.

LupUlinus.

Lupulina, Linn.

Obliquus, Fab.

Fuscus, Haw., var.

Angulum, Haw., var.

Nebulosus, Haw., var.

Sylvinus.

Sylvina, Linn.

Lupulina, Hub.
VELLEDA.

Velleda, Esp.

Mappa, Don.

Carnus, Steph., var.

Humuli.

Humuli, Linn.

COCLIOPIDAE, Gn.

I.I MACODES, Lal.

AsElLUS.

Asellus, W. $\boldsymbol{V}$.

Testudo.

Testudo, $W \cdot V$

o Bufo, Fab.

Funalis, Don.

PROCRIDA, Gn.

PROCRIS, Fab.

INO, Leach.

Statices.

Statices, Linn.

Globularia. $50 / 64$

Globularim, $H u b$.

Serson then

ZYGAENIDAE, Gn.

ZYGANA, Fab.

ANTHROCERA, Scop.

Minos.

Minos, $W . V$

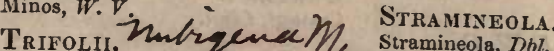

Trifolir. Lubrgena/ha Stramineola, Dbl. (præc. var.?)

Loti, Haw. $\sum$ U 62,50 Quadra.

LONICERE.

Loniceræe, Esp.

Filipendulate.

Filipendulve, Linn.

Hippocrepidis, Steph., var.

-

\section{NOLIDE, Gn.}

NOLA, Leach.

Cucullatella.

Cucullatella, Linn.

Cristulatis.

Cristulalis, $H u b$.

Strigulalis, Haw.

Strigula.

Strigula, $W . V$.

Monachalis, Haw.

Centonalis the 59

LITHOSIDAE, Gn.

NUDARIA, Haw.

Senex.

Senex, $H u b$.

Rotunda, Haw.

Mundana.

Munidana, Linn.

Hemerobia, Hub., var.

SETINA, Steph.

IRRORELLA.

Irrorella, Linn.

Roscida, Steph., var.
CALligenia, Dup.

Miniata.

Miniata, Forst.

Rosea, Fab.

LITHOSIA, Fab.

Mesomella.

Mesomella, Linn.

Eborina, $\boldsymbol{H} u b$.

Muscerda.

Muscerda, $H u b$.

AUre Ola.

Aureola, $H u b$.

Pygmála.

Pygmæola, $\mathrm{Dbl}$.

Pallifrons, Zeller.

Luteola, Mus. Cat. (non Hub.)

Helveoua.

Helveola, Och.

Complanula.

Complanula, $B d v$.

Complana, Haw.

Complana. $\backslash$ S 50

Complana, Linn.

Depressa, Steph.

Griseor.A.

Griseola, $H u b$.

Plumbeolata, Steph.

Gilveola, Steph., var.

Quadra, Linn.

RUBRICOLLIS.

Rubricollis, Linn.

EULEPIA, Curt.

EMYDIA, $B d v$.

Grammica.

Grammica, Linn.

Cribrum.

Cribrum, I,inn.

EUCHELIDAE, Gn.

DEIOPEIA, Curt.

Pulchella.

Pulchella, Linn.

EUCHELIA, $B d v$.

J ACOBEE, Linn.

CALLIMORPHA, Lat.

Dominula.

Dominula, Linn.

CHELONIDAE, Gn. EUTHEMONIA, Steph.

Russula.

\& Russula, Linn.

$\checkmark$ Sannio, Linn. 
CHELONIA, Lat.

ARCTIA, Steph.

Plantaginis.

Plantaginis, Linn.

Hospita, W. V., var.

Caja.

Caja, Linn;

Villica.

Villica, Linn.

ARCTIA, $B d v$.

SPILOSOMA, Curt.

Fuliginosa.

Fuliginosa, Linn.

MENDICA.

Mendica, Linn.

\section{LUBRICI'E DA.}

Lubricipeda, Linn.

Menthastri.

Menthastri, $\boldsymbol{W} . \boldsymbol{V}$.

URTIC 压.

Urtica, Esp.

Papyratia, Marsh.

\section{LIPARIDAE, $\mathrm{Gn}$.}

LIPARIS, Och.

Chrysorthea.

Chrysorrhæa, Linn.

Phæorrhæa, Haw.

Auriflua, Steph.

Auriflua.

Auriflua, Fab.

Chrysorrhæa, Haw.
SALICIS.

Salicis, Linn.

Dispar.

Dispar, Linn.

Monacha.

Monacha, Linn.

ORGYIA, Och.

PUdibunda.

Pudibunda, Linn.

Fascelina.

Fascelina, Linn.

Coenosa.

Cœnosa, Hub.

Gonostigma.

Gonostigma, Linn.

Antiqua.

Antiqua, Linn.

DEMAS, Steph.

Coryli.

Coryli, Iiun.

BOMBYCIDAE. TRICHIURA, Steph.

Crategi.

Cratægi, Linn.

PCECILOCAMPA, Steph.

Populi.

Populi, Linn.

\section{ERIOGASTER, $G n$.}

LANESTRIS.

Lanestris, Linn.
BOMBYX, Linn.

CLISIOCA MPA, Curt.

LASIOCAMPA, Steph.

Neustria.

Neustria, Linn.

Bilineatus, Haw., var.

Castrensis.

Castrensis, Linn.

RuBI.

Rubi, Linn.

Quercus.

Quercus, Linn.

Roboris, Steph., var.

Calluna.

Callunæ, Palmer (præc. var.?)

TRIFOLII.

Trifolii, $W . V$.

Medicaginis, Bork., var.

ODONESTIS, Germ.

Potatoria.

Potatoria, Linn.

LASIOCAMPA, Lat.

GASTROPACHA, Curt.

Quercifolia.

Quercifolia, Linn.

Alnifolia, Och., var.

ILICIFOLIA.

Ilicifolia, Linn.

ENDROMIS, Och.

VERSICOLORA.

Versicolora, Linn.

SATURNIA, Schr.

Carpini.

Carpini, $B$ ork.

Pavonia, var. minor, Linn.

GEOMETRA, Linn.

URAPTERYDAE, Gn. OURAPTERYX, Leach.

Sambucata.

Sambucata, Linn.

ENNOMIDAE, Gn.

EPIONE, Dup.

Vespertaria.

Vespertaria, Linn.

Parallelaria, $w \cdot v$.

A picaria.

Apiciaria, $W . V$.

Vespertaria, Don. (non Linn.)

ADVENARIA.

Advenaria, $H_{u b \text {; }}$
RUMIA, Dup.

Crategata.

Cratægata, Linn.

VENILIA, Dup.

Maculata.

Maculata, Lin

Quadrimaculata, Hatch., var.

ANGERONA, Dup.

Prunaria.

Prunaria, Linn.

Sordidata, Naturf., var.

Dimidiata, Fab., var.

Corylata, Esp., var.
METROCAMPA, Lat.

Margaritata.

Margaritata, Linn.

ELLOPIA, $\boldsymbol{T} \boldsymbol{r}$.

Fasciaria.

Fusciaria, Linn.

Prosapiaria, Linn.

EURYMENE, Dup.

DolobraRia.

Dolobraria, Linn.

PERICALLIA, Steph.

Syringaria.

Syringaria, Linn. 
SELENIA, $H u b$.

ILlUNARIA.

Illunaria, $H u b$.

Ustularia, Don.

Juliaria, Haw. (var. æst.)

LCNARIA.

Lunaria, $W, V$.

Sublunaria, Steph., var.

Delunaria, Hub. (var. æst.)

I LLUSTRARIA.

Illustraria, $\mathrm{Hub}$.

Delunaria, Sleph. Mus. (var. æst.)

ODONTOPERA, Sleph.

Bidentata.

Bidentata, Linn.

Deutaria, $H u b$.

CROCALLIS, $T r$.

Elinguaria.

Elinguaria, Linn.

ENNOMOS, $T r$.

\section{AlNiaria.}

Alniaria, Linn.

Tiliaria.

Tiliaria, $H u b$.

Alniaria, Haw.

Canaria, Sleph., var.

Fuscantaria.

Fuscantaria, Haw.

Carpinaria, Haw. (non Hub.)

Erosaria

Erosaria, $W . V$.

Quercinaria, Bork.

Ochraria, Steph., var.

Angularia.

Angularia, $W . V$

o Tiliaria, Haw.

Carpinaria, Steph., var.

Quercinaria, Steph., var.

Quercaria, Steph., var.

HIMERA, Dup.

Pennaria.

Pennaria, Linn.

Bifidaria, Haw., var.

$A M P H Y D A S Y D A E, G$. PHIGALIA, Dup.

Pilosaria.

Pilosaria, $W, V$.

Pedaria, Fab.

NYSSIA, Dup.

ZoNARIA.

Zonaria, $W . V$.

HISPIDARIA.

Hispidaria, $W, V$

Ursularia, Don.

Tauaria, Neum.

\section{BISTON, Leach.}

Hirtaria.

Hirtaria, Iinn.

Congeneraria, Haw., var.

Funaria, Haw., var.
AMPHYDASIS, $T r$.

Prodromaria.

Prodromaria, $W . \nabla$.

Betularia.

Betularia, Linn.

\section{BOARMIDAE, Gn.} HEMEROPHILA, Steph.

Abruptaria.

A bruptaria, Thun.

Petrificata, $\boldsymbol{H u b}$.

Suberaria, Don.

\section{CLEORA, Curt.}

Viduaria.

Viduaria, $W . V$.

Grabratia.

Glabraria, $H u b$.

Teneraria, $H u b$.

Lichenaria.

Lichenaria, $W . V$.

BOARMIA, $T r$.

RePandata.

Repandata, Linn.

Conversaria, Hub., var.

Destrigaria, Steph., var.

Muraria, Curt., var.

Rhombotdaria.

Rhomboiduria, $W . V$.

Consobrinaria, Haw., var.

Australaria, Curt., var.

Fimbriaria, Steph, var.

Abietaria.

Abietaria, $W . V$.

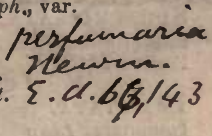

Cinctaria.

Cinctaria, $W . V$.

Roboraria.

Roboraria, $W . V$.

Consortaria.

Consortaria, Fab.

\section{TEPHROSIA, $B d v$.}

\section{Consonaria.}

Consonaria, $H u b$.

Tetragouaria, Haw.

Crepuscularia.

Crepuscularia, $W . V$.

Abietaria, Haw., var.

Laricaria, Dbl. Cat.

Biundularia.

Biundularia, Esp. (præc. var.?)

Crepuscularia, Haw. (var. ver.)

Crepuscularia, var., $G n$.

Extersaria.

Extersaria, $H u b$.

Punctulata.

I'unctulata, $W . V$.
GNOPHOS, $T r$.

Obscurata.

Obscurata, $W . V$

Pullaria, Hub. 145., Haw., var. (uon $W, V$.)

Serotinaria, Haw., var.

Dilucidaria, Steph., var.

DASYDIA, $G n$.

Obfuscata.

()bfuscata, $W \cdot \nabla$.

Canaria, $H u b .344$.

Limosaria, Hub. 360.

Operaria, Curt. (ron Hub)

PSODOS, $T r$.

Trepidaria.

Trepidaria, $\boldsymbol{H} u b$.

MNIOPHILA, $B d v$.

Cineraria.

Cineraria, $W . V$.

Corticaria, Dup.

BOLETOBIDAE, Gn. BOLETOBIA, Bdv.

Fuliginaria.

Fuliginaria, Linn.

Carbonaria, $W . V$

GEOMETRIDAE, Gn. PSEUDOPTERPNA, Hub.

Cytisaria.

Cytisaria, $W . V$.

Prasinaria, Fab.

GEOMETRA, Linn.

Papilionaria.

Papilionaria, Linn.

Smarag Daria.

Smaragdaria, $H u b$.

NEMORIA, Hub.

Viridata.

Viridata, Linn.

Porrinaria, Zell., var.

Cloraria, Steph., var.

IODIS, $H u b$.

VerNaria.

Vernaria, Livin.

Lucidata, Don.

LACTEARIA.

Lactearia, Linn.

Eruginuria, $\boldsymbol{H u b}$.

Putataria, Haw., Steph., non Linn.

PHORODESMA, Bdv.

Bajularia.

Bajularia, $W . V$.

Ditaria, Fab.

HEMITHEA, Dup.

Thymiaria.

Thymiaria, Linn.

Astivaria, Brahm. 
EPHYRIDAE, $\mathrm{Gn}$. EPHYRA, Dup.

Porata.

Porata, Linn.?

Punctaria, $W . V$.

Ucellaria, Haw.

Punctaria.

Punctaria, Linn.

Subangularia, Haw., var.

Trilinearia.

Trilinearia, Bork.

Linearia, Hub.

OMicronaria.

Omicronaria, $W . V$.

Orbicularia.

Orbicularia, $H u b$.

Pendulairia.

Pendularia, Linn.

ACIDALID AE, Gn.

HYRIA, Steph.

Aurolraria.

Auroraria, $G n$.

Auroralis, $W . V$.

Variegata, $F a b$.

ASTHENA, Hub.

J,UTEATA.

Luteata, $\boldsymbol{W} . \boldsymbol{V}$.

Flavostrigata, Don.

Candidata.

Candidata, $W . V$.

Immutata, $F a b$.

Candidulata, Huw.

Sylvata.

Sylvata, $W$. $\Gamma$.

Testaceata, Don.

Blomeraria.

Blomeraria, $\mathrm{Dbl}$.

Blomeri, Curt.

Pulchraria, Evers.

EUPISTERIA, $B d v$.

Heparata.

Heparata, $W . V$.

VENUSIA, Curt.

Ca mbricaria.

Cambricaria, $Q n$.

Cambrica, Curt.

Erutaria, $\boldsymbol{B} d v$.

\section{ACIDALIA, $T r$.}

\section{Ochrata.}

('chrata, Scop.

Pallidaria, Dup.

Perochraria, Pbt-(non Fisch.)

Rubricata. $s, \alpha>1.81$

Rubricata, $W . \nabla$.

Scutulata.

Scutulata, $W \cdot V$.

Lividata, Huw.
Bisetata.

Bisetata, Bork.

Reversaria, Dup.

Dilulata, Haw.

Fimbriolata, Steph., var.

Cinereata, Steph., var.

Trigeminata.

Trigeminata, Haw.

Reversata, Tr.?

Bisetaria, Dup.

Contiguaria.

Contiguaria, Hub. (non Haw.)

Rusticata.

Rusticata, $W . V$.

INORNATA.

Inornata, Haw.

Suffusata, Tr., var.?

Degeneraria.

Degeneraria, $H u b$.

Emarginata.

Emarginata, Linn.

Rumigerata, Don.

Dimidiata, Haw.

TIMANDRA, Dup.

Amataria.

Amataria, Linn.

Osseata. Osseata, $W . V$ Ldifutarege thet

Holosericatä.

Holosericata, Dup. . .

INCANARIA.

Incanaria, $H u b$.

Virgularia, $H u b$.

Circellata.

Circellata, $G n$.

Obsoletaria, Dbl.Cat. (non Rawb.)

Ornata.

Urnata, Scop.

Promutata.

Promututa, $G n$.

Immutata, $W \cdot V$. (non Linn.)

Incanata, Haw. (non Linn.)

Contiguaria, Haw. (nou Hub.)

Siraminata.

Straminata, $T r$.

Marginepunctata, Steph.?

placs cumi

SubSERICEATA.

Subsericeata, Haw. $\mathrm{Haw} 2 \mathrm{2} / 30$
Perfluaria, Bdv.

IMMUTATA.

Immutata, Linn.

5.064144

Caspitaria, Bdv.

Reмutata. Stiquarea th A. Stiquerea

Remutata, $\mathrm{H} u b$.

Flos-lactata, Haw.

Lactata, Haw., var.

Sublactata, Haw., var.

Fumata.

Fumata, Steph.

Commutata, Frey.

Nitidaria, $\boldsymbol{B} d v$.

Strigilata.

Strigilata, $W . V$.

Exemptaria, Hub. Verz.

Variegata, Steph.

Prataria, $\boldsymbol{B} d v$.

IMITARIA.

Imitaria, $H u b$.

$C A B E R I D \not E, \mathrm{Gn}$.

CABERA, $T r$.

Pusaria.

Pusaria, Linn.

RotUN DARIA.

Rotundaria, Haw.

Continaria, Frey.

Exanthemaria.

Exanthemaria, Scop.

Striaria, $H u b$.

Arenosaria, Haw., var.

Approximaria, Haw., var.

CORYCIA, Dup.

Temerata.

Temerata, $W . V$.

Punctata, Fab.

Nubeculata, Haw.

Taminata.

Taminata, $W . V$.

Bimaculata, Fab.

AIEUCIS, $G n$.

Pictaria.

Pictaria, Curt.

Emutaria.

Emutaria, $H u b$.

Subroseata, Haw.

Aversata.

Aversata, Linn

Remutata, Linn.

Lividata, Linn., var.

MACARIDAE, $\mathrm{Gn}$. MACARIA, Curt.

Alternata.

Alternata, $W . V$.

Notata.

Notata, Linn.

Liturata.

Liturata, Linn.

HALIA, Dup.

WAVARIA.

Wavaria, Linn.

\section{FIDONIDAE, Gn.} STRENIA, Dup.

Clathrata.

Clathrata, Linn.

Radiata, IIaw., var.

Retata, Hau., var. 


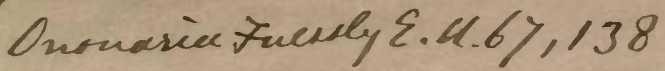

PANAGRA, $\boldsymbol{G}$.

Petraria.

Petraria, Hub.

NUMERIA, Dup.

Pulveraria.

Pulveraria, Linn.

SCODIONA, $B d v$.

Belgiaria.

Belgiaria, $H u b$.

Favillacearia, Hub., 139.

Mediopunctaria, Don.

\section{SELIDOSEMA, Led.}

Plumaria.

Plumaria, $W . V$.

Ericetaria, Vill.

\section{FIDONIA, $\boldsymbol{T} \boldsymbol{r}$.}

Carbonaria.

Carbonaria, Einn.

Picearia, Hub.

\section{A tomaria.}

Atomaria, Linn.

Glarearia, Haw., var.

Roseidaria, Haw., var.

\section{Piniaria.}

Piniaria, Linn.

\& Tiliaria, Linn.

Pinetaria.

Pinetaria, $H u b .130$.

Quiuquaria, Hb.-Fey., 516.

Sylvaria, Curt.

Brunneata, Steph.

Conspicuata.

Conspicuata, $W . V$.

Limbaria, $\boldsymbol{F} a b$.

MINOA, $T r$.

EUPHORBIATA.

Euphorbiata, $W, \nabla_{0}$

SCORIA, Steph.

DEaLbata.

Dealbata, Linn.

STERRHA, $H u b$.

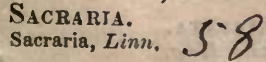

ASPILATES, $\boldsymbol{T} r$.

Strigillaria.

Strigillaria, $H u b$.

Respersaria, $\mathrm{Hub}$.

Inæquaria, Haw., var.

Citraria.

Citraria, Hub.

Gilvaria.

Gilvaria, $W . V$.
ZERENID AE, Gn. ABRAXAS, Leach.

Grossulariata. Grossulariata, Linn.

Ulmata.

Ulmata, $F a b$.

LIGDIA, $\boldsymbol{G} n$.

Adustata.

Adustata, $W . \nabla$.

LOMASPILIS, $H u b$.

Marginata.

Marginata, Linn.

Pollutaria, Hub, var.

\section{LIGIDAE, Gn.}

PACHYCNEMIA, Steph.

Hippocastanaria.

Hippocastanaria, Hub.

\section{$H Y B E R N I D A E, \mathrm{Gn}$.}

HYBERNIA, Lat.

Rupicapraria.

Rupicapraria, $W, \nabla$.

Primaria, Haw.

LEUCOPHEARIA.

Leucophearia, $W . V$.

Nigricaria, Hub., Haw., var.

A urantiaria.

Aurantiaria, $H u b$.

o Prosapiaria, Haw.

\& Incompletaria, Hau.

Progemaria.

Progemmaria, $H u b$.

o' Stictaria, Haw.

\& Luctuaria, Haw.

Leucopbearia, + , Wood, 459 .

Defollarid.

Defoliaria, Linn.

ANISOPTERYX, Steph.

Escularia.

Escularia, $W . V$.

\& Apteraria, Haw.

\section{LARENTIDAE, Gn.}

CHEIMATOBIA, Steph.

Brumata.

Brumata, Linn.

Boreata.

Boreata, $\mathrm{Hub}$.

OPORABIA, Steph.

Dilutata.

Dilutata, $W . V$.

Fimbriata, Haw., var.

Neglectata, Steph., var.

Fuligram maria.

Filigrammaria, Herr.-Schf.

Autumnaria, Dbl. Cat. vur.? non $B d v$.
LARENTIA, $T r$

Didymata.

Didymata, Linn.

Scabrata, $H u b$.

Multistrigaria.

Multistrigaria, Haw.

Cesiata.

Cæsiata, $W, V$.

Infrequentata, Hau.

Ruficinctata.

Ruficinctata, $\dot{G} \boldsymbol{n}$.

Flavicinctata, Sleph, (non $H u b$.)

Salicata.

Sulicata, $H u b$.

I,atentaria, Curt.

Olivata.

Olivata, $W$. $V$.

Pectinitaria.

Pectinitaria, Fuess.

Miaria, $H u b$.

FMMELESIA, Steph.

Affinitata.

Affinitata, steph.

Rivulata, Dbl. Cat.

Alchemillata.

Alchemillata, Linn.

Rivulata, $W$. $V$.

Hydraria, Dbl. Cat. (non $T r$.)

Albulata.

Albulata, $W . V$.

Niveata, Steph, 291.

Decolorata.

Decolorata, $H u b$.

Tenrata.

Tæniata, Sleph.

Aletaria, Herr.-Sch.

UNIFASCIATA.

Unifasciata, Haw

Bifasciata, Havo.

Aquilaria, Herr.s.Sch.

Fricetata.

Ericetata, Curt.

Braniata.

Blandiata, $W . \nabla$.

Trigonata, Steph.

EUPITHECIA, Curt.

Venosata.

Venosata, $\mathrm{Fab}$.

Decussata, Don.

Consignata.

Consignata, Bork.

LinaRIaTA.

Linariata, $W$. $\nabla$.

Pulchellata.

Pulchellata, Steph.

Centaureata.

Centaureata, $W . V$.

Succenturiata.

Succenturiata, $\operatorname{Linn}$.

Disparata. Hub., var.?

Oxydata, $\boldsymbol{T} r_{.}$, var.?

Subfulvata, Haw., var.?

Cognata, Sleph., var.? 


\section{Campannlate HSSMI/4Z}

Subumbrata.

Subumbrata, $W . V$.

Piperata, Steph.

Obrutaria, Herr.-Sch.

Pernotata.

Pernotata, Gn.

Plumeeolata.

Plumbeolata, Haw.

Begrandaria, $\boldsymbol{B} d v$.

Haworthiata.

Haworthiata, $D b l$.

Plumbeolata, var. $\beta$, Haus.

Pygm eata.

Pygmæata, $\boldsymbol{H} u b$.

Pygmrata, $H u b$.
Palustraria, $D b l$.
HelveticARIA. 58

Reductaria, $B d v$.

Helveticaria, $B d v$. Crecenth $728 y$

Cxiguata.

Satyrata, Hub. $2 \mathrm{CG}_{3}, 21$ Exiguata, $\mathrm{H} u \mathrm{~b}$

Callunaria, Stainton, var.

Albipunctata, Hawo?

Egrnaria.

Egenaria, Herr.-Sch.

Castigata.

Castigata, Haw.

Nebulata, Steph.

Austerata, $H u b ., 45$.

Valerianata, $B d v$

Residuata, $\boldsymbol{H} u b$., var, ?

I W T PIMPINELLaTA.

D, 94 Pimpinellata, $H u b$. Rufifasciata, Haw.

VIMINata. $50<7$

Viminata, $\mathrm{Dbl}$. 2 l 3 Coronata.

Pusillata.

Pusillata, $W$. $r$.

IrRiguATA.

Irriguata, $H u b$.

Variegata, Haw.

Denotata.

Denotata, $\boldsymbol{H} u b$.

Pimpinellata, Herr.-Sch.

Pimpinellata, Herr.-Sch.

Innotio

INDIGATA.

Indigata, $H u b$.

Constrictata.

Constrictata, $G n$.

Distinctaria, Herr.-SCh.?

Nanata.

Nanata, $H u b$.

Angustata, Haw.

Subnotata.

Subnotata, $H u b$.

Simpliciata, Haw.

Vulgata.

Vulgata, Haw.

Austerata, Herr.-Sch.

Subfuscata, Haw., var.

- Expallidata.

Expallidata, $G n$.

Absynthiata.

Absynthiata, Linn.

o Elongata, Haw.
Trimaculata, Haw

Lanceolaria, Dbl. Cat, var.

Sobrinata.

Sobrinata, $\mathrm{Hub}$.

Lævigata, Haw.

Expressaria, Kerr,-Sch., var.?

TOgata.

Togata, $H u b$.

Pumilata.

Pumilata, $H u b$.

Strobilata, Steph.

Bistrigata, Haw.

Coronata, $H u b$.

V-ata, Haw.

\section{Rectangulata.}

Rectangulata, Linn.

Subærata, Steph., var.

Nigrosericeata, Haw., var,

Debiriata.

Debiliata, Hub.

COLLIX, $G n$.

Sparsata.

Sparsata, $H u b$.

LOBOPHORA, Curt.

\section{Sexalata.}

Sexalata, $H u b$.

Hexapterata.

Hexapterata, $W . V$

Zonata, Bork, var.

Viretata.

Viretata, $H u b$.

Trinotata, Don.

Lobulata.

Lobulata, $F u b$.

Dentistrigata, Haw.

Costæestrigata. Haw.

Rupestrata, Steph.

Polycommata.

Polycommata, $W$. $V$.
THERA, Steph.

JUNIPERATA.

Juniperata, Linn.

Simulat $\mathrm{T}$.

Simulata, $H u b$.

Coniferata, Curt.

Geneata, Feisth., var.

FaRtata.

Farrata, W. $V$

Trivtrigaria, Dor.

Muntrata, Havo.

Obeliscata, $H u b$., जar.

Iutvata, ofteph, var.

Simułarta, Bdv., var.

Firmata.

Firmata, $H u b$.

YPSIPETES, Steph.

RUBERATA.

Ruberata, Frey.

Literata, Don.?

IMPLUVIATA.

Impluviata, $W . V$.

Elutata.

Elutata, $W . V$.

MELANTHIA, Dup.

Rubiginata.

Rubiginata, $W . V$.

Plumbata, Curt., var.

Ocellata.

Ocellata, Linn.

A lbicillata.

Albicillata, Linn.

MELANIPPE, Dup.

Hastata.

Hastata, Linn.

Tristata.

Tristata, Linn.

Procellata.

Procellata, $W . V$.

UNANGULATA.

Unangulata, $H$ aw

Amniculata, $H u b$. ?

Rivata.

Rivata, $\boldsymbol{H} u b$.

Sylvaticata, Haw.

Subtristata.

Subtristata, Haw.

Rivata, var., $G n$.

Degenerata, Haw., var.

Montanata.

Montanata, $W . V$.

Implicata, Haw.

Galiata.

Galiata, $W . V$.

Unilobata, Haw., var.

Quadriannulata, Haw., var,

Fluctuata.

Fluctuata, $\operatorname{Linn}$.

Costovata, Haw., var. 
ANTICLEA, Steph.

Sivuata.

Sinuata, $W \cdot \nabla$.

RUBIDATA.

Rubidata, $W . V$.

Badiata.

Badiata, $W$. $r$.

Derivata.

Derivata, $W . V$.

Berberata.

Berberata, $W, V$.

COREMIA, $G n$.

Munitata.

Munitata, $\boldsymbol{H} u \boldsymbol{b}$.

Propugnata.

Propugnata, $W . V$.

Ferrugata.

Ferrugata, Linn.

Salicaria, Haw., var.

Unidentaria, Haw., var.?

QUADRIFAsCIARIA.

Quadrifasciaria, Linn.

Ligustraria, $W . V$.

CAM PTOGRAMMA, St.

Bilineata.

Bilineata, Linn.

Fluviata.

Fluviata, $H u b$. $\delta$

Gemmata, $\boldsymbol{H} u b$, 웅

Angustata, Haw. $\$$

PHIBALAPTERYX, $s t$.

Tersata.

Tersata, $\boldsymbol{W} . \vec{V}$.

LAPIDATA.

Lapidata, Hub.

LigNaTA.

Lignata, $H u b$.

Lineataria, Don.

Polygrammata.

Polygrammata, Bork.

Conjunctaria, Lederer, var.?

Viralbata.

Vitalbata, $W, \cdot$.

\section{SCOTOSIA, Steph.}

\section{Dubitata.}

Dubitata, Linn.

Cinereata, Steph., var.
Vetulata.

Vetulata, $\boldsymbol{W} . V$.

Rhamnata.

Rhamnata, $W . V$.

Certata.

Certata, Hub.

Cervinata, Haw.

UNdUlata.

Undulata, Lipnn.

CIDARIA, $T r$.

Psitracata.

Psittacata, $W . V$.

Mrata.

Miata, Linn.

Coraciata, Hub.

Picata.

Picata, $R u b$.

Biangulata, Haw.

Corytata.

Corylata, Thunb.

Ruptata, $H u b$.

Albocrenuta, Curt., var.

SagitTata.

Sagittata, Fab.

Comitata, I $u$ ub.

Russata.

Russata, $W . V$.

Centum-notata, Fab.

Comma-notata, Haw., var.

Perfuscata, Haw., var.

ImvanatA.

Immanata, Hau.

Marmorata, Haw., var.

Russata, var., Gn.

Suffumata.

Suffumata, $W . \nabla$.

Piceata, S'leph., var.

Silaceata.

Silaceata, $W . V$.

Prunata.

Prunata, Linn.

Ribesiaria, $B d v$.

Testata.

Testata, Linn.

Achatinata, $\mathrm{H} u b$.

Populata.

Populata, Bork.

Fulvata.

Fulvata, Forst.

Sociata, Fab.

Pyraliata.

Pyraliata, Bork.

Populata, Linn.?

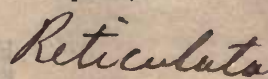

WV

¿ab2,110
Dotata.

Dotata, Linn.

Marmorata, Hub.

Spinachiata, Haw.

PELURGA, Hub.

Comitata.

Comitata, Linn.

Chenopodiata, $W . V$.

EUBOLIDAE, Gn.

EUBOLIA, Dup.

Cervinaria.

Cervinaria, $W . V$.

Clavaria, Haze.

Mensuraria.

Mensuraria, $W$. $V$.

Chenopodiata, Linn.?

Palumbaria.

Palumbaria, $W$. $V$.

Bipunetaria.

Bipunctaria, $\boldsymbol{W} . V$.

Lineolata.

Lineolata, $W . V$.

Heceniata W.V.S.

CARSIA, Hub.

IMBUTATA.

Imbutata, $H u b$.

ANAITIS, Dup.

Plagiata.

Playiata, Linn.

LITHOSTEGE, $H u b$.

Niviaria.

Nivearia, $W$. $F$.

Clarkiata, Curt.

CHESIAS, $\boldsymbol{T r}$.

Spartiata.

Spartiata, Fab.

Obliquarta.

Obliquaria, $W . V$.

Rufuta, Fab.

Bomlycata, Don.

$$
\text { SIONIDIE, Gn. }
$$

TANAGRA, Dup.

CHAROPHYLLATA.

Chærophyllata, Linn. 


\section{DREPANULÆE, $G n$.}

\begin{tabular}{|c|c|c|}
\hline DREPANULIDAE, Gn. & $\begin{array}{l}\text { SICULA. } \\
\text { Sicula, } W . V \text {. }\end{array}$ & $\begin{array}{l}\text { UNGUICULA. } \\
\text { Unguicula, Hub. }\end{array}$ \\
\hline LACERTULA. & $\begin{array}{l}\text { Falcula. } \\
\text { Falcula, } W . V \text {. }\end{array}$ & CILIX, Leach. \\
\hline Lacertinasia, Linn. & $\begin{array}{l}\text { HaMuLA. } \\
\text { Hamula, } W . v \text {. }\end{array}$ & $\begin{array}{l}\text { SPINULA. } \\
\text { Spinula, } W . V . \\
\text { Compressa, Fab. }\end{array}$ \\
\hline
\end{tabular}

\section{PSEUDO-BOMBYCES, $G n$.}

\author{
DICRANURIDAE, Gn. \\ DICRANURA, Lat. \\ CERURA, Schr. \\ Bicuspis. \\ Bicuspis, Bork. \\ Furcula. \\ Furcula, Linn. \\ Bicuspis, Steph. (non Bork.) \\ Latifuscia, Curt., vas. \\ BIFIDA. \\ Bifida, Hub. \\ Arcuata, Steph. \\ Fuscinula, Steph. \\ VINULA. \\ Vinula, Linn. \\ Erminea, Steph. (non $H u b$,)
}

STAUROPUS, Germ.

Fagi.

Fagi, Linn.

PETASIA, Stepk.

Cassinea.

Cassinea, Fab.

Nubeculosa.

Nubeculosa, Esp.
PYGARIDAE, Gn. PYG正RA, Och.

Bucephat.A.

Bucephala, Linn.

Clostera, Hoff.

Cuntula.

Curtula, Linn.

Reclusa.

Reclusa, $W . V .0$ \& 60,128 NOTODONTIDAE, Gn.

GLUPHISIA, $B d v$.

Crenata.

Crenata, Esp.

P'TILOPHORA, Steph.

Plumigera.

Plumigera, $W . V$.

Variegata, Steph.

PTII.ODONTIS, Steph.

Palpina.

Palpina, Linn.

NOTODONTA, Och.

Camelina.

Camelina, Linn.

Cuculuina.

Cucullina, $W . V$.
Carmelita.

Carmelitu, Esp.

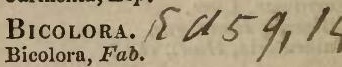

DICTEA.

Dictæa, Linn.

Dictent DEs.

Dictæoides, Esp.

Dromedarius.

Dromedarius, Linn.

Dromedarulus, Haw', var.

Perfusca, Steph. var.

Tritophus.

Tritophus, $W \cdot V$.

Perfuscus, Haw.?

Ziczac.

Ziczac, Linn.

TrEPIDA.

Trepida, Fab.

Chaonia.

Chaonia, $W$. $V$.

Roboris, Fab.

Dodonea.

Dodonea, $W . V$.

Querneus, Haw., var.

DILOBA, $B d v$.

C ARU LEOCE PHaLA.

Cæruleocephala, Linn.

\section{NOCTUE, Linn.}

TRIFID AE, Gn. BOMBYCIFORME'S. NOCTUO-BOMB YCID E, Bdv. THYATIRA, Och.

Disisa.

Derasa, Linn.
Batis.

Batis, Linn.

CYMATOPHORA, $T$ ir.

Duplaris.

Duplaris, I,inn.

Bipuncta, Bork.
Fluctuosa.

Fluctuosa, Hub.

Diruta.

Diluta, $W, V$.

Or.

Or, W. V.

Flavicornis, Haw. 
Ocularis.

Ocularis, Jinn.

Octogena, Esp.

Octogesima, $H u b$.

Flavicoris.

Flavicornis, Linn.?

Luteicornis, Haw.

\section{RiDENS.}

\section{Ridens, $F a b$.}

Chrysoceras, Beckwith.

Xanthoceros, Bork.

BRYOPIIILIDAE, Gn.

BRYOPHILA, Tr.

Glaniffera.

Glandifera, W. V.

Lichenis, Fub.

Par, Hub., var.

PERla.

Perla, $W . V$.

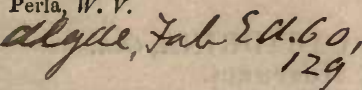

BO. $B$ YCOID E, Bdv.

DIPHTHERA, Och.

Orion.

Orion, $E s p$.

Ruuica, Haw.

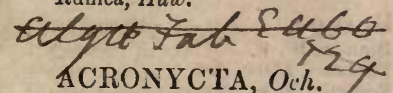

Tridens.

Tridens, $W . V$.

Psi.

Psi, Linn.

Cuspis, Steph. var.

IEPORINA.

Leporina, Linn.

Brady porina, $T r_{\text {., var. }}$

AcERIS.

Aceris, Linn,

Infuscata, Haw., var.

Megacepha La.

MegacepLala, $W \cdot V$.

Strigosa.

Stricosa, $F a b$.

Favillacea, Naturf.

AlNI.

Alni, Linn.?

Ligustri.

Ligustri, $W . V$.

Corouulu, Huw., var.

Rumicis.

Rumicis, Linn.

Salicis, Curt.

Auricona.

Auricoma, $W, V$.

Sımilis, Haw, var.

Menyanthidis, Hau , var.

Menyantulds.

Menyanthidis, Esp.

Mrric 无.

Myrica, Gu.

Euphorbiw, Dbl. Cat.
SIMYRA, Och.

VENOSA.

Venosa, Bork:

Degener, $H u b$.

Atomina, Haw.

GENUINAE, G॥. LEUCANIDAE, Gn.

SYNIA, Dup.

Musculosa.

Musculosa, $I$ inb.

Nervosa, Steph.

LEUCANIA, $O c h$.

Conigera.

Conigera, $I V . r$.

Viteli.INA.

Vitellina, $H u b$.

Turca.

Turca, Linn.

Lithahgyria.

Lithargyria, $E s p$.

o Auargyria, Dup. $\sum$ tram

Obsolera. I \& 60,129

Obsoleta, $H u$.

LITTORALIS.

Littoralis, Curt.

Pudorina.

Pudorina, $W . V$.

Comma.

Comma, Linn.

Straminea.

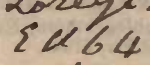

Straminea, $T r$.
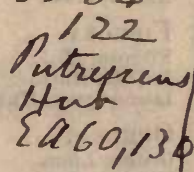

Impura.

Impura, $H u b$.

Punctina, Haw., var.

Pallens.

Pallens, Jinn.

Rufescens, Haw., var.

Areuuta, Steph., var.

Suffusa, Steph., var.

Ochracea, Steph., var.

Phragmitidis.

Phragmitidis, $H u b$.

MELIANA, Curt.

Flammea.

Flammea, Curt.

Arundinicola, Dul. Cat.

SENTA, Steph.

ULVA.

Ulvæ, $H u b$.

Sericea, Curt.

Bipunctata, Haw., var.

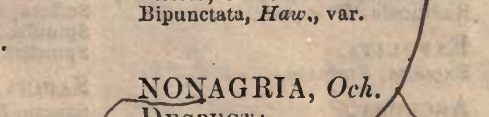

DEspeCTA.

Tespectr, $T r$.

Kufa, Hax.?

Lineola, Stcph, var.
Fulva.

Fulva, $H a b$.

Pygmina, Haw.

Fluxa, Steph.

Pullida, Sleph, var.

Neurica, Steph., var.

Concolor.

Concolor, $G n$.

Extrema, Hub.?

HELLMANNI.

Hellmanni, Evers.

Neurica.

Neurica, $H u b$.

Hessii, Boisd., vac

Geminipuncta.

Geminipuncta, Hatch.

Paludicola, Hub.

Cansa.

Camwe, Och.

TYPHE.

Typhæ, Esp.

Arundinis, $\boldsymbol{F} a b$.

I.U'Yosa.

Lutosa, $H u b$.

Crassicornis, Haw.

Bathyerga, Frey.

Pilicornis, Haw., var.

in Elymi is $\mathcal{L} U 62$,

GOR'TYNA, Och.

Flavago.

Flavugo, $W . V$.

()chraccago, Haw,

Lappæ, Don.

HYDRECIA, $G n$.

Nictitans.

Nictitans, Linn.

Auricula, Don.

Lucens, Herr.-Sch., var.?

Petasitis.

Petasitis, Dbl.

Vindelicia, Frey.

Micacea.

Micaceu, Esp.

Cypriaca, Hub.

AXYI.IA, Hub.

Putris.

Putris, Linn.

XYLOPHASIA, Steph.

\section{Rurea.}

Rurea, Fab.

Combusta, $H u b$., var.

LiTHOXYLEA.

Lithoxylea, $W \cdot \boldsymbol{V}$.

SUbLUST Ris.

Sublustris, Esp.

Musicalis, Dup.

Polyonon.

Polyodon, Linn.

Hepatica.

Hepatica, Linn.

Characterea, $H u b$.

Epomidion, Haw. 


\section{Scolopacina.
Scolopacina, Esp. \\ Abbreviata, Hau \\ DIPTERY\&IA, Steph. \\ Pinastri. \\ Piuastri, Linn \\ XYLOM GES, $G n$. \\ Cosspicillaris. \\ Conspicillatis, $W . V$. \\ Leuconota Don. \\ APOR 0 PHYLA, $G n$. \\ Australis. \\ Austral /s, $B d v$. \\ Pascuea, Curt. \\ LAPHYGMA, $\boldsymbol{G}$. \\ Exidua. \\ Exigua, Hub. \\ NEURIA, $G n$. \\ Salonarize. \\ Sap nariæ, Esp.}

HELIOPHOBUS, $B d v$.

Popularis.

Poyularis, Fab.

Typicoides, Don.

HISPIDA.

H spida, Hub.

qHAREAS, Steph.

GRAMINIS.

framinis, Linn.

Hibernicus, Curt.

PACHETRA, Gn.

LEUCOPHAA.

eucophæa, $W . V$.

CERIGO, Sleph.

Cytuerea.

Cytherea, Fab.

Texta, Esp.

\section{I.UPERINA, $B d v$.}

TESTACEA.

Testacea, $W . V$.

Unce, Haw., var.

Lunato-strigata, Haw., var.

X-yotata, II aw., var.

Cu 2 w

Cespitis, $W . V$.

Autumualis, Curt.

$$
\text { in }
$$

\section{MAMESTRA, $O i h$.}

\section{ABJECTA.}

A bjecta, $H u b$.

Nigricans, Steph.

A NCEPS.

Anceps, $H u b$.

Infesta, Och.

Aliena, Dup.

Contigua, Haw.

\section{Albicolon.}

Albicolon, $H u b$.

Furva.

Furva, $W, V$.

Brassice.

Brassicæ, Linn.

Albidilinea, Haw., var.

Pensicania.

Persicariæ, Linn.

APAMEA, Orh.

Basilinea.

Basilinea, $W . \nabla$.

Connexa.

Conuexa, Bork.

Elota, Hub.

Gemina.

Gemina, $H u b$,

Furva, Steph. (non W. V.)

Remissa, Hub., var.

Ublonga, Haw., var.

(Hoscura, Haw., var.

UNANIMIS.

Unanimis, $H u b$.?

Secalina, Haw.

Ophiogramar.

Ophiogramma, Esp.

Biloba, Haw.

Fibrosa.

Fibrosa, Hub.

Lunina, Haw.

Leucostigna, $H u b$. var.

Oculea.

()culea, Linn.

Didyma, Bork.

Lugens, Haw., var.

I-nigel, Haw., var.

Furca, Haw., var.

Rava, Haw., var.

MIANA, Steph.

Strigilis.

Strigilis, Linn.

Latruncula, $W . V$.

Aithiops, Haw., var.

Fasciuncula.

Fasciuncula, Haw

Rubeuniula, Donzel.

Strigilis, var., Gn.

IATEROSA.

Literosa, Haw.

Erratricula, $H u b$,

Suffuruncula, $T^{\prime} \%$.

Furuncula.

Furuncula, $W . V$.

Humerulis, Haw.

'ferminalis, Haw., var.

Rufuncula, Haw., vas.

Expolita.

Expolitu, Stainton.

Arcuosia.

Arcuosa, Haw.

Minima, Haw.

Duponchelii, $\mathbf{B} d v$.
CELANA, Steph.

HawORTHII.

Haworthii, Curt.

Luncea, Steph., var.

Tripuncta, Curt., var.

Erupta, Freyer.

Hibernica, Steph., var.

CARADRINID E, Gn. GRAMMESIA, Steph.

Trilinea.

Triliuea, $W . V$.

bilinea, $H u b$, var.

ACOS.METIA, Steph.

Caliginosa.

Caliginosa, $\mathrm{Hub}$.

Lutesceus, Haw.

CARADRINA, Och.

\section{MORPHEUS.}

Morpheus, Naturf.

Sepii, Steph.

Alsines.

Alsines, Bork.

Sordida, Haw.

BlaNdA.

Blauda, $W . V$.

Taraxaci, Herr.-Sch.

Redacta, Haw., var.

Ambigua, Steph., var.

Inplexa, Steph., var.

Iævis, Steph., var.

Cubicularis.

Cubicularis, $W, V$.

Superstes, Steph.

NOCTUIDAE, Gn.

RUSINA, Steph.

TENEBRosa.

Tenebrosa, $H u b$.

Phæus, Haw.

q Ubsoletissima, Haw.

AGROTIS; Och.

Valligera.

Valligera, $W . V$.

Clavis, Esp.

Sagittiferus, Haw.

Puta.

Puta, Hub.

Lignosa, God., var.

Radius, Haw.

Tadiola, steph.

Suffusa.

Suffusa, $W . V$.

Spinula, Esp., Don.

spinifera, Haw.

Saucia.

Saucia, $H u b$.

Áqua, Hub., var.

Margaritosa, Huw.

Majuscula, Huw. 
Segetum.

Segetuin, $W . V$.

Catsenatus, Haw., var.

Pectiuatus, Haw., var.

Monileus, Haw., var.

Spinulus, Haw., var.

Connexus, Haw., var.

Corticeus, Haw., var.

Venosus, Havo, var.

subatratus, Haw., var.

nigricornutus, Haw., var.

LUNIGERA.

Lunigera, Steph.

Exclamationis.

Exclamationis, Linn.

\& Picea, Haw.

Plaga, Steph., var.

Corticed.

Corticea, $\boldsymbol{W} . \boldsymbol{V}$.

$\checkmark$ Clavigerus, Haw.

Subfiscus, Haw.

\section{Cinerea.}

Cinerea, $W . V$.

Denticulatus, Haw.

RIP压.

Ripæ, $H u b$.

Nebulosa, Steph.

Cursoria.

Cursoria, Bork.

Venosa, Steph., var.

Nigricans.

Nigrieans, Linn.

Fumosa, $\boldsymbol{W}$. $\boldsymbol{V}$.

Ruris, Haw., var.

Sordida, Haw., var.

Obeliscata, Haw, var.

Duhia, Haw., var.

Marshallana, West., var.

Tinicici.

Tritici, Linn.

Lineolata, Haw., var.

Albilinea, Haw., var.

Valligera, Haw., var.

Cuneigera, Steph., var.

Sagittifera, Steph., vur.

Ucellina, Steph, var.

AqUILINA.

Aquilina, $W . V$.

Vitta, Steph., var.

Hortorum, Steph., var,

Obelisca.

Obelisca, $W$. $V$.

Agathina.

Aguthina, Dup.

Albimacula, steph.

Lcucographa, Steph.

Porphyrea.

Porphyrea, W. $V$.

Ericæ, $\mathrm{Hau}$.

Precox.

P'ræcox, Linn.

Ravida.

Ravida, $W . V$

Crassa, Huw.

Pyrophild.

Pyrophila, $W . V$.

LUCERNEA.

Luceruea, Linn.

lienizera, steph.

Cataleucu, Bdv.
Ashivorthit.

Ashworthii, $D b l$.

TRIPHENA, Och.

Janthina.

Jantbina, $W \cdot V$.

Finbria.

Fimbria, Linn.

INTERJECTA.

Interjecta, $H u b$.

Subsequa.

Subsequa, $W . V$.

OnBona.

Orbona, Fab.

Pronuba.

Pronuba, Linn.

Iunuba, $\Gamma r$., var.

NOCTUA, Gn.

Gianeosa.

Glareosa, Esp.

Hebraica, Hub.

I-geminum, Dup.

Depuncta.

Depuncta, Linn.

Augur.

Augur, Fab.

Plecta.

Plecta. Flammatra Ixsta BILIs.

C-Nigrum.

C-nigrum, Linn.

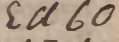

Ditrapezium.

Ditrapezium, $I I u b$.

Tristigma, $T r$.

Triangulum.

Triangulum, $O c h$.

Rhombordea.

Rhomboidea, $T r$.

Stigmatica, $H u b$.

Tristigma, Steph.

Brunnea.

Brunuea, $W . V$.

Festiva.

Festiva, $W \cdot V$.

Subrufa, Haw., var.

Conflua.

Conflua, $T r$. (præc, var, bor.?)

Dahlit.

Dahlii, $H u b$.

Erythrocephala, $\mathrm{Haw}_{0}$

Subrosfa.

Subrosea, Steph.

RuBI.

Rubi, Tieneg.

Bella, Bork,

lunicea, Haw.

UмBROSA.

Umbrosa, Hub.

Sex-strigala, Haw.

BAJA.

Baja, $W^{5} \cdot V$.

Alpina.
Sobriva.

Sobrina, $\boldsymbol{B} d v$.

Neg LECTA.

Neglecta, $H u b$.

Lævis, Haw.

Xanthographa.

Xanthogrupha, $W \cdot V$.

T'etragous, Haw.

ORTHOSIDAE, Gn. TRACHEA; $G n$.

Piniperda.

Piniperda, Esp.

\section{PACHNOBIA, $G n$.}

Alpina, Westwood.

Carnica, Heer.

\section{TENIOCAMPA, Gn.}

GoTHICA.

Gothica, Linn.

Levcogra pha.

Letcographa, $W . T$.

Rubricosa.

Rubricosa, $W . V$.

Mista, Hub., var.

Rufa, Haw., var.

Instabilis, $W . V$.

Fuscatus, Haw.

Nebulosus, Haw, var.

Subsetaceus, Haw, var.

Angusta, Haw., var.

Opima.

Opima, Haw.

Populiet.

Populeti, $\boldsymbol{F a b}$

Subplumbeus, Haw.

Gracilis, Steph.

Stabilis.

Stabilis, $\boldsymbol{W} . \boldsymbol{V}$.

Cerasi, Fab., Haw.

Junctus, $\mathrm{Haw}_{\text {, }}$ var.

Rufaniulata, Haw., var,

Gracilis.

Gracilis, $W . V$.

Spursus, Haw.

Pallida, Haw., var.

Miniosa.

Miniosa, $W . V$.

Munda.

Munda, W. $V$.

Bimaculatus, Haw.

Geminatus, Haw.

Cruda.

Cruda, $\boldsymbol{W}, \boldsymbol{V}$.

Nanus, Haw.

Pusilla, Haw.

ORTHOSIA, Och.

Suspecta.

Suspecta, $H_{u b}$.

Congener, $\boldsymbol{H} u b .-G_{j} c y$. 
Yrsilon.

Ypsilon, $W$. $V$.

Fissipuncta, Haw.

Lota.

Lota, Linn.

Macilenta.

Macilenta, $H u b$.

Flaviliuea, Huw.

\section{A.NCHOCELIS, $G n$.}

\section{RuFina.}

Rutina, Linn.

Helvola, Linn., Haw.

Pistacina.

Pistacina, $W$. $V$.

Lineola, Dun., var.

Ferrea, Haw., var.

Lunosa.

Lunosa, Haw.

Agrotoides, $G n$.

Subjecta, Dup.

Litura.

Litura, Linn.

CERASTIS, Och.

GI.ÆA, Steph.

VACCINII.

Vaccinii, Linn.

Polita, Haw., var.

Spadicea.

Spadicea, $\boldsymbol{W} \cdot \boldsymbol{V}$.

Subuigra, Haw., var.

Ertithocephala.

Erythrocephala, $\boldsymbol{W} . \boldsymbol{V}$.

Glabra, $W . V$. , var.

SCOPELOSOMA, Curt.

Satellitia.

Satellitia, Linn.

DASYCAMPA, $G n$.

RUBIGINEA.

Rubiginea, $W . V$.

HOPORINA, Bdv.

Croceago.

Croceago, $W . V$.

Aurautiago, Don.

XANTHIA, Och.

Citrago.

Citrago, Linn.

Cerago.

Cerago, $W . V$.

Fulvago, Haw.

Gilvago, Haw., var.

Silago.

Silago, $H u b$.

Flavago, $\mathrm{Fab}$.

lubago, Dun.

Aurago.

Aurago, $W, V_{\text {: }}$
Gilinago.

Gilvago, $E s p$.

Ferruginea.

Ferruginea, $W . V$.

Macilenta, Haw.

CIRRCEDIA, $G n$.

Xeramielina.

Xerampelina, $\boldsymbol{H u b}$.

Centrago, Haw.

COSMID 2 , Gn.

TETHEA, Och.

Subtusa.

Subtusa, $W . V$.

Retusa.

Retusa, Linn.

Gracilis, Haw., var.

EUPERIA, $G n$.

Fulvago.

Fulvago, $W . V$.

\section{DICYCLA, $G n$.}

Oo.

Oo, Linn.

Renago, Haw.

COSMIA, Och.

Trapezina.

Trapezina, Linn.

Pyralina.

Pyraliua, $W . V$.

Diffinis.

Difinis, Liun.

Affinis.

Affinis, Linn.

HADENIDAE, Gn. EREMOBIA, Steph. II.ARUS, Bdv.

Ochroleuca.

Uchroleuca, $W . V$.

Citriua, Don.

DIAN'THACIA, $B d v$.

Carpophaga.

Carpophaga, Bork.

Ochracea, Haw. , var.

Capsincola.

Capsincola, W. V.

Cucubali.

Cucubali, W. $V$.

Rivularis, Fub.

Consiersa.

Conspersa, $W \cdot V$.

Compta, Haw.

?

\section{HECATERA, $G n$.}

DYSODEA.

1)ysodea, $W . V$

Ranunculina, Huu.
Serena.

Serena, $W . V$.

Par, Don.

POLIA, Och.

Chi.

Chi, Linn.

Ulivacea, Steph., var.

Flavocincta.

Flavocincta, Linn.

Vigrocineta $O$

DASYPOLIA, $G n$.

Templi.

Templi, Thunb.

EPUNDA, Dup.

Lutulenta.

Lutulenta, $W . V$.

Fusca, $H a w$.

Consimilis, Steph., var.

Sedi, $B d v$., var.

Nigra.

Nigra, Haw.

Eithiops, Och.

VIMINAlis.

Viminalis, Fab.

Scripta, Hub., Huw.

LICHENEA.

Lichenea, $H u b$.

VALERIA, Germ.

Orelagina.

Uleagina, $W . V$.

MISELIA, Och.

OXYaCANTHA:.

Oxyacauthæ, Linn.

AGRIOPIS, Blv.

Aprilina.

Aprilina, Linn.

PHLOGOPHORA, $O$

Meticulosa.

Meticulosa, Linn.

EMPYREA.

Empyrea, $H u b$.

EUPLEXIA, Steph.

Lucipara.

Lucipara, Linn.

APLECTA, $G n$.

Herbida.

Herbida, $W$. $V$.

Mixta, Ifaw.

Occulta.

Occulta, Linn.

Nebclosa.

Nebulosa, $\mathrm{Tr}$.

Grandis, Don., Hau. 
Tincta.

Tineta, bork.

Argentina, Haw.

Advena.

Advena, $W . V$.

HADENA, Och.

Satura.

Satura, $W V$.

Assmints: Assimilis, Duto

Adusta.

Adusta, Esp.

Valida, $H u b$.

Duplex, Haw.

Satura, Steph., var.

Proted.

Protea, $W . V$.

Seladouia, Huw.

Grauca.

Glauea, Kleem.

Lappo, Dup., var.

Dentina.

Dentina, $W . V$.

Plebeja, Haw.

Leucostigma, Haw.

$\underset{\text { Peregrina, } T r}{\text { Peragina. }} 5459,1 \mathrm{~kb}$

Chenopodir.

Chenopodii, W. V.

Atriplicis.

Atriplicis, Linn,

Suasa.

Suasa, $W, V$.

Dens-canis, Hau.

Oreracea.

Oleracea, Linn.

Pisi.

Pisi, Linn.

Splendens, Steph, var.

Thalassina.

Thalassina, Naturf.

Achates, $H u b$.

Humerulis, Haw.

Contigua.

Contigua, $W . V$.

Dives, $H a w$.

Geniste.

Geniste, Bork.

W-Latinuın, Hufu,?

Rectilinea, Haw. (non Esp.)

Rectilinea.

Rectilinea, Esp.

XYLINIDAE, Gn. XYLOCA. IPA, Gn.

Lithoriza.

Lithoriza, Bork.

CLOAN'THA, Bdv.

Perspicillaris.

Perspicillaris, Linn.
Solmagivis.

Solidagiuis, Hub.

CALOCAMPA, Steph.

Vetusta.

Vetusta, $\boldsymbol{H u b}$.

Exoleta.

Exoleta, Linn.

XYLINA, Och.

Rhizolitha.

Rhizolitha, $W \cdot V$.

Lambda, Huw.

SFimbrunnea.

Semibrunnea, Haw.

Uculata, Germ.

Petrificata.

Petrificata, $W . V$.

Caporme

CUCULLIA, Ock.
Verbasci.

Verbasci, Linn.

Scrophulariet

Scrophularix, $W \cdot V$.

LYCHNITIS.

Lychnitis, Rambur.

Asteris.

Asteris, $W . V$.

GNaPhaI.II.

Gnaphalii, $H u b$.

Absinthir.

Absinthii, Linn.

Chanomille.

Chamomillæ, $W . V$.

Fissina, Haw.

Chrysauthemi, $H u b$, var.

UMBRATICA.

Umbratica, Linn.

ot Tanaceti, Steph. (non W. V.)

\& Lactuce, Steph. (non W. V.)

$\$$ Lucifuga, steph. (non $W . V$.)

HELIOTHIDA, Gn.

HELIOTHIS, Och.

MaRginata.

Marginata, $\mathrm{Fab}$.

Peltigera.

Peltigera, W. $V$.

Straininea, Don.

Armigera.

Armigera, $I u b$.

Dipsacea.

Dipsacea, Linn.

ANARTA, Och.

Melanopa.

Melanopa, T'uunb,

Vidua, $T r$.
Cordigera.

Cordigera, Thunb.

Myrtilli.

Myrtilli, Linn.

HELIODES, $G n$.

A RBUTt.

Arhuti, Fab.

Heliaca, $W . V$.

MINORES. ACONTIDAE, Gn. AGROPHILA, $B d v$.

Sulphuralis.

Sulphüralis, $W . V$.

ACONTIA, Och.

Lucteosa.

Luctuosa, $W . V$.

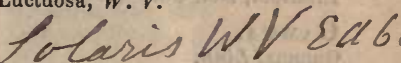

ERASTRIDAE, Gn.

ERASTRIA, Och.

Venustula.

Venıstula, $K u b$.

Fuscula.

Fuscula, $W . V$.

BANKIA, $G n$.

Argentula.

Argentula, Esp.

Bankiana, $F a b$.

ANTHOPHILIDA, Gn. HYDRELIA, $\boldsymbol{G} n$.

UNCA.

Unca, Linn.

MICRA, $G n$.

Ostrina.

Ustrina, $H u b$.

PHALAENOIDAE, Gn.

BREPHOS, Och.

Parthenias.

Parthenias, Linn.

Notha, Haw., Cur't.

Notha.

Notha, Hub.

Partheuias, Hau, , Curt.

QUADRIFID AE VARIEGATAE, Gn. PLUSIDAE, Bdv. ABROSTOLA, Och.

URTICA:

Urticie, Hub. 
Triplasia.

Triplasia, Linn.

Orichalcea.

Orichalcea, $\mathrm{Fab}$.

AE rifera, Sowerby.

Chrisitis.

Chrysitis, Linn.

Bractea.

Bractea, $W$. $\boldsymbol{V}$.

Festuce.

Festucæ, Linn.

IoTA.

Iota, Linn.

Percontationis, Sleph.

V-AUREUM.

$\nabla$-aureum, $G n$.

Iota, var. pulchrina, Haw.

Iota, Steph.

Inscripta, $\mathrm{Dbl}$. Cat.

Ga M M.

Gamma, Linn.

INTFRROGATIONIS.

Interrogationis, $H u b$.

Verticulates 5 .

GONOPTERIDAE, Gn.

GONOPTERA, Lat.

Libatrix.

Libatrix, Linn.
PLUSIA, Och.

INTRUSAE. AMPHIPYRIDAE, Gn. AMPHIPYRA, Och.

Pyramidea.

Pyramidea, Linn.

Tragopogonis.

Tragopogonis, Linn.

Tetra, Haw.

MANIA, $T r$.

Typica.

Typica, Linn.

Maura.

Maura, Linn.

TOXOCAMPID AE, $\mathrm{Gn}$. TOXOCAMPA, $G n$.

\section{Pastinum.}

Pastinum, $T r$.

Lusoria, Haw.

Craced $\mathrm{Wl}$

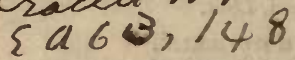

STILBIDAE, Gn.

STILBIA, Steph.

A Nomala.

Anomala, Haw.

Hybridata, $H u b$.

Lathyri, $B d v$.

\section{DELTOIDES, Lat.}

HYPENIDAE, H.-Sch. MADOPA, Steph.

Salicalis.

Salicalis, $W . V$.

HYPENA, Sch.

Proboscidalis.

Proboscidalis, Linn.

Rostralis.

Rostralis, Linn.

Vittatus, Haw., var.

Palpalis, Steph., var.

Crassalis.

Crassalis, $\mathrm{Fab}$.

Achatalis, II ub.

\section{HYPENODES, $G n$.}

A r. Bistrigalis.

Albistrigalis, Haw.

Nonalis, Gn., MSS.

Costestrigalis.

Costæestrigalis, Steph.

Leucopteralis, Gn., MSS.

Acuminalis, Herr.-Sch.

SCHRANKIA, Herr.-Sch.

TURFOSALIS.

Turfosalis, Wock.

Humidalis, $\mathrm{Dbl}$.

HER.MINIDAE, Dup. RIVUIA, $G_{n}$.

Sericealis.

Sericealis, $W . V$.
LIMBATAE. ₹व CATOCALIDAE, Gn. CATOCALA, Och.

\section{Fraxini.}

Fraxini, Linn.

Nupta.

Nupta, Linn.

Promissa.

Promissa, $W . V$.

Conjuncta, Steph., var.

Sponsa.

Sponsa, Linn.

SERPENTINAE, Gn. $O P H I U S I D A E, \mathrm{Gn}$. OPHIODES.

\section{LunaRIS.}

Lunaris, $W, V$.

EUCLIDIDAE, Gn. EUCLIDIA, Och.

MI.

Mi, Linn.

Glyphica.

Glyphica, Linn.

POAPHILIDAE, Gn. PHYTOMETRA, Haw. ENEA.

Enea, $W . V$.

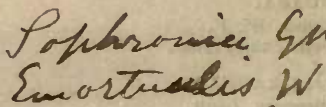

HERMINIA, Lat. $\sum a \succeq$

DERIVALIS.

Derivalis, $H u b$.

Emurtualis, Haw.

Barbalts.

Barbalis, Linn.

Tarsipennai.Is.

Tarsipennalis, $T r$.

Tarsicrinalis, Haw.

Grisealis.

Grisedlis, $W . V$.

Nemoralis, Fab., Haw.

Cribralis.

Cribralis, $H u b$.

\section{AVENTI屈.}

AVENTIDA, Gn.

AVENTIA, Dup.

Flexula.

Flexula, F'ub. 


\section{PYRALIDES.}

SQUAMOSA, Gn.

ODONTIDA, Gu. ODONTIA, Dup.

Dentalis.

Dentalis, $W . V$.

\section{PULVERULENT F, Gn PYRALIDAR, G॥.}

PYKALIS, Linn.

Fimbrialis.

Fimbrialis, $W . V$.

Costalis, Fab.

Farinalis.

Farinalis, Linn.

Glaucinalis.

Glaucinalis, Linn.

Nitidalis, $H u b$.

AGLOSSA, Lat.

Pinguinalis.

Pinguinalis, Linn.

Streatfieldii, Curt., var.

Cuprealis.

Cuprealis, $\mathrm{Hub}$.

Capreolatus, Haw.

CLEDEOBIDAE, Dup. CLEDEOBIA, Steph.

A ngtistalis.

Angustalis, $W . V$.

Boinbycatus, Haw.

\section{LURIDAE, Gn.} ENNYCHIDA, Gn. PYRAUSTA, Schr.

Punicealis.

Punicealis, $W . V$.

Porphyralis, Haw.

Purpuralis.

Purpuralis, Linn.

Ostrinalis.

Ostrinalis, $\mathrm{Hub}$.

Punicealis, Haw.

RHODARIA, $\boldsymbol{G n}$.

Sanguinalis.

Sanguinalis, Linn.

HERBULA, $\boldsymbol{G} n$.

Cespitalis.

Cespitalis, $W . V$.

Sordidulis, $\dot{H} u \dot{b}$.
ENNYCHIA, $T r$.

Cingulalis.

Cingulalis, Linn.

Anguivalis.

Anguinalis, $\boldsymbol{H} u b$.

Octomaculalis.

Octomaculalis, Iiinn.

Guttalis, $W . V$.

Atrulis, $F u b$.

\section{$A S O P I D A E, \mathrm{Gn}$.}

AGROTERA, Schr.

Nemoralis.

Nemoralis, Scop.

Erosalis, Fab.

\section{ENDOTRICHA, Zell.}

Flam Mealis.

Flammealis, $W . V$.

\section{STENIADAE, Gn.}

DIASEMIA, Steph.

J.ITERALIS.

Literalis, Scop.

Argentalis, $F a b$.

Ramburialis.

Ramburialis, Dup.

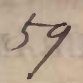

NASCIA, Curt.

Cilialis.

Cilialis, $H u b$.

Acutalis, Evers., Gn.

STENIA, $G_{n}$.

Punctalis.

Punctalis, $W . V$.

Longipedalis, Dale.

IY DROCA MPIDAE, Gn CATACLYSTA, $H .-5 \mathrm{sch}$.

LEMNALIS.

Lemnalis, Linn.

\& Uliginata, $F a b$.

PARAPONYX, Sieph.

Stratiotalis.

Stratiotalis, Linn.

HYDROCAMPA, Lat.

Nympiealis.

Nymphæalis, Linn.

o Potomogalis, Linn.

Stagnalis.

Stagnalis, Don.

Nymplasealis, Haw.
BOTYDA, Gn.

BO'TYS, Lat.

LUPULINALIS.

Lupulinalis, $C l k$.

Silacealis, $\boldsymbol{H} u b$.

\section{Pandalis.}

Pandalis, $H \pi b$.

Terminalis, Haw.

Angustalis, Haw.

Flavalis.

Flavalis, $W . V$.

Hyalinalis.

Hyalinalis, $\boldsymbol{H} u b$.

VERTICALIS.

Verticalis, $W$. $V$.

LANCEALIS.

Lancealis, $W . V$.

Glabralis, Hub. 117.

Longalis, Haw.

Fuscalis.

Fuscalis, $W . V$.

Cineralis, Haw.

Fimbrialis, Steph., var.

Terrealis.

Terrealis, $\boldsymbol{T} r$.

Borealis, Curt.

Asinatis.

Asinalis, $\boldsymbol{H} u b$.

Urticalis.

Urticalis, Linn.

EBULEA, Gn.

Crocealis.

Crocealis, $\mathrm{Tr}$.

Ochrealis, Hub., 146.

Verbascalis, Wood.

Verbascalis.

Verbascalis, $\boldsymbol{W} \cdot \boldsymbol{V}$.

Arcualis, $H u b$., 80.

Sambucalis.

Sambucalis, $W . V$.

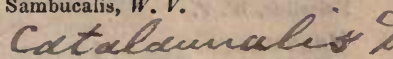

PIONEA, $\boldsymbol{G} n$.

Forficalis.

Forficalis, Linn.

Margarita Lis.

Margaritalis, $\mathrm{Fab}$.

Erucalis, $\boldsymbol{H} u \boldsymbol{b}$.

Stramentalis.

Stramentalis, $\boldsymbol{H} u b$.

\section{SPILODES, $G \boldsymbol{n}$.}

Sricticalis.

Sticticalis, Linn.

Fuscalis, $K u b, 45$

Tetragonalis, Huw. 
Palealis.

Palealis, W. $V$.

Selenalis, Hub., 177.

Cinctalis.

Cinctalis, $T$ 'r.

Limbalis, Haw.

Verticalis, Linn. Mus. 2060

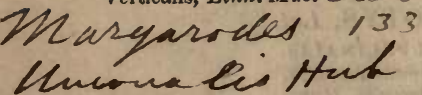

SCOPULA, Schr., Gn.

Alpinalis.

Alpinalis, $W . V ., G n$.

Uliginosalis, Curt., var.

LuTEA LIS.

Lutealis, Haw., Gn., Hub. 145.

Pascualis, Lienig.

Etialis, Gn. MSS.

Olivalis.

Olivalis, $W . V$.

Nivealis, Haw.

Prunalis.

Prunalis, $W . V$.

Nebulalis, Haw.

Ferrugalis.

Ferrugalis, $\boldsymbol{H u b}$.

Decre pitalis.

Decrepitalis, Herr..Sch.
MECYNA, $G n$.

Polygonalis.

Polygonalis, $\boldsymbol{H} u b$.

Diversalis, $T r$., var.

\section{PLICATAE, Gn. SCOPARIDAE, Gn. STENOPTERYX, $G n$. \\ HYBRIDalis. \\ Hybridalis, $H u b$.}

SCOPARIA, Haw., Gn. EUDOREA, Curt.

Ambigualis.

Ambigualis, $T r$.

Dubitalis, Haw., Steph:

Cembralis.

Cembralis, $G n$.

Cembræ, Haw.

Subfusca, Haw., var.

Pyralalis.

Pyralalis, $W$. $V$.

Tristrigella, Steph., var.

Tristrigella, Steph., var. tella Zell PARALIs.

Muralis

Muralis, Gn.

Murana, Curt.

Lineolalis.

Lineolalis, fin.

Lineolea, Cur't

3 asists

uluella CRAMBITES.
Uliginosellus.

Uliginosellus, $Z$ ell.

Cretaceellus, Dale, MSS.

Furcatellus.

Furcatellus, Zett.

Lapponicellus, Dup.

Radiellus, Curt. (non Hub.)

Margaritellus.

Margaritellus, $\boldsymbol{H} u b$.

Pinetellus.

Pinetellus, Linn.

Couchella, $H u b$.

Latistriellus.

Latistria, Haw.

Leachellus, $Z$ inck.

Guenéellus, Dup.

Perielalus.

Perlellus, Scop.

Argentea, Hau:

Dealbellus, Steph., var.

Arbustea, Haw., var.

Arbustoruin, Steph., var.

Argyrea, Haw., Steph., var.

WARRING TONELLUS.

Warringtonellus, Zell. (præc. var.?) Lithargy rellus, Steph, Ill.

LiTHARTERELLUS. Lithargyrellus, $K u b$.
Mercuralis.

Mercurella, Linn.

Frequentella, Stuinton.

Concinnella, Curt, var.?

Crategalis.

Cratægella, $H u b$.

Pusilla, West., var.

Resinalis.

Resinea, Haw.

Delunella, Gn., olim.

Pheolevcatis.

Portlandica, Dale?

Truncicola lis.

Truncicolella, Stainton.

Coarctalis.

Coarctalis, Zell.

Angustea, Curt.

A Tomalis.

Atomalis, $\mathrm{Dbl}$.

Gracilatis.

Gracilalis, $D b l$.

Parella, Zell.

Alpina, Dale.

Pallidulalis.

Pallidulalis, $G n$.

Pallida, Curt.

Ertznerialis, Herr.-sch.?
Phæoleuca, Zell.?

\section{Adipellus, Tr., Inb. V'erz.}

H A M ELUS.

Hamellus, Thunb.

Ensigerellus, $\boldsymbol{H} u l$ ).

Pascuellus.

Pascuellus, Linn.
Selaselius.

Selasellus, Hub., Steph. Obtusellus, Steph.

Tristellus.

Tristellus, $W . V$

Aquilella, Hub.52, Steph.

Culmella, Fub., Steph., var.

Paleellus, Hub., Steph, var.

Petrificellus, Steph., var.

Fuscelinellus, Steph., var.

Nigristriellus, Steph, var.

Pedriolellus.

Pedriolellus, Dup.

INQUINATEILUS.

Inquinatellus, $W . V$.

Rorea, Haw.

Luteellus, Steph. (non $W . V$.

Contamineluus.

Contaminellus, $\mathrm{Hub}$.,

Aridella, Thunb.?

Geniculei.lus.

Geniculea, Haw.

Immistellus, $H u b$.

Inquinatellus, Steph.

Angulatellus, Dup.

Culmelaus.

Culmellus, Linn.

Straminella, Hub.

Striga, Huw. 
Chrysonucheltus.

Chrysonuchellus, Scop.

Campea, Haw.

Rorellus.

Rorellus, Linn.

Craterella, Scop.

Linetella, Fab.

Chrysouuchella, Hub.

Cassentilielus.

Cassentiliellus, Mann.

Hortueleus.

Hortuellus, $\mathrm{Hub}$.

Cespitellus, Hub., var,?

Palodelius.

Paludellus, $\mathrm{Hub}$.

Cbilo obtusellus, Stainton.

Ocellea How

$C H I L I D A E, \mathrm{Gn}$.

CHILO, Zinck.

Cicatricelius.

Cicatricellus, $H u b$.

Strigellus, $\boldsymbol{T} r$.

Phragmitellus.

Phragmitellus, $H u b$.

\% Rhombea, Haw.

\& Gigantea, Haw.

\section{SCHOENOBIUS, Dup.}

\section{Forficellus,}

Forficellus, Thunb.

ơ Consortella, $H u b$.

\& Lanreolella, Hub.

Hirta, Haw., var. $\delta$.

Fumea, Haw., var. $\delta$.

Caudea, Haw., var. $\$$.

Mucronellus.

Mucronellus, Scop.

\& Acuminella, $\mathrm{Hub}$.

Gigantellus.

Gigantellus, $W, \nabla$.

\& Punctigerellus, Curt., Steph.

PHYCIDAE, Gn.

ANERASTIA, Hub., Zell. ARAXES, Steph.

\section{LOTELLA.}

Lotella, $H u b$

Miniosella, $\boldsymbol{T r}$.

Farrella.

Farrella, Curt. (præc, var.?)

ILITHYIA, Lat.

Carnella.

Carnella, Linn.

Sanguinella, Hub, var.

MYELOPHILA, Tr., Gn.

\section{Cribrella.}

Cribrella, Hub.

Cribrum, $W . V$.

Cardui, Haw., Steph.
HOMEOSOMA, Curt.

LOTRIA, Gn.

Sin UElla.

Sinuella, Fab.

Gemina, Haw.

NimbeLLd.

Nimbella, Dupe Jacichla V/2. Nebulei.la: $i a->1 \lg 2$ Nebulella, Hub

Eruvient Fenecenis

Eluviella.

of Binrevella, Steph.

\& Nebulella, Steph.

NYCTEGRETES, Zell.

Achatinella. Achatinella, $\mathrm{Hub}$.

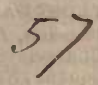

\section{EPHESTIA, $G n$.}

\section{Elutella.}

Elutella, $H u b$. text, $C r n$.

Rufa, Haw., var.

Angusta, Haw., var.

Ficella.

Ficella, Dougl.

Semirufella.

Semirufa, Haw.

Pinguedinella.

Pinguedinella, $G n$.

Pinguis, Haw.

Artemisier.la.

Artemisiella, Steph.

CRYPTOBLABES, Zell.

Bistrigella.

Bistrigella, Haw.

Rutilella, Fisch.?

PLODIA, $G n$.

INTERPUNCTELLA.

Interpunctella, $H u b$.

NEPHOPTERYX, Zell.

ANGUSTELLA.

Angustella, $\boldsymbol{H u b}$.

GYMNANCYCLA, Zell.

\section{Canella.}

Canella, $W . V$

Depositella, Zinck.

\section{PHYCIS, Fab.}

Betulella.

Betulr, Goetz.

Obtusella, Zinck.

Tristrigella, Steph.

Christella, Frey.

Holoserjceellu, $F$ isch.
Carbonariella.

Carbonariella, Fisch. Fusca, Haw.?

ADELPHELLA.

Adelphella, Fisch.

Hostilis, Steph.

Dilutella.

Dilutella, $H u b$.

Adornatella, Dup.

Subornatella, Z ell.

Serpyletorum, Z Z ell.

$\&$ Obscura, Steph.

Ornatella.

Ornatella, $W . V$.

Criptella, $\boldsymbol{L u b}$., Curt.

ABietella. Otowelea

A bietella, $W . V$.

Decuriella, $\boldsymbol{H} u \boldsymbol{u}$.

Roborella.

Roborella, $T$. $T$.

Spissicella, Fab.

Spissicornis, Haw.

Legatea, Haw., var.

Cristea, Haw., var.

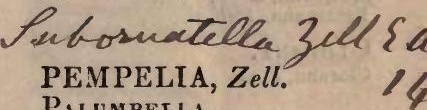

Palumbella.

Palumbella, $W . V$.

Contuburnella, Hub., Hav.

Cinerea, West., var.

RODOPHEA, $\boldsymbol{G}$.

Formosella.

Formosa, Haw.

Perfluella, Zinck.

Consociella.

Consociella, $\mathrm{Hub}$.

Fascia, Haw., var.?

Advenelta.

Advenella, Zinck.

Palumbea, Haw.

Hhenella, Steph.

Recurvella, Gn., var.?

Marmorella.

Marmorea, Haw.

Epelydella, Fisch.? G̛n.

Suavella.

Suavella, Zinck.

Porpbyrea, Curt., Steph.

TuMinella.

Tumidella, Zinck.

Verrucella, Hub,

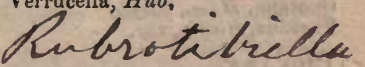

ONCOCERA, Steph.

HYPOCHALCIA, Zell.

Ahenella.

Ahenella, $W . V$.

Obscuratus; Haw.

En neellu, Hub., var.

Tetrix, Haw., var.

Bistrigella, Dup., var.

Fuliginella, Dup , vur. 
MELIA, Steph., Gn. APHOMIA, Step. Mus. Ct.

\section{Sociella.}

Sociella, Linn.

\& Colonella, Linn.

Tribunella, $W . V$.

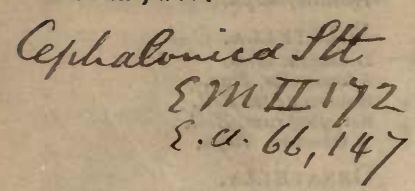

\author{
$C Y M B I D A A, \mathrm{Gn}$. \\ HALIAS, $T r$, $G n$. \\ Prasinana. \\ Prasinana, Linn. \\ Fagana, Fab. \\ Quercana. \\ Quercana, $\boldsymbol{W}$. $\boldsymbol{V}$. \\ Prasinana, Fab. \\ Clorana. \\ Clorana, Linn.
}

TORTRICIDAE, Gn. SARROTHRIPA, Curt.

Revayana.

Revayana, $W . V$.

Rivagana, $\mathrm{Fab}$.

Ilicana, Fab., var.

Afzelianus, Wood.

Punctana, Hub., var.

Ramosana, Hub., var.

Stonanus, Curt., var.

Ramosana, Dup.

Ramulanus, Cur't, var.

Afzeliana, Steph., var.

Ilicanus, Wood.

Lathamianus, Steph., var.

Dilutana, Hub., var.

Undulana, $H u b$. var.

Degenerana, $H u b$, var.

\section{TORTRIX, Linn.}

LOZOT ENIA, Steph.

Pyrastrana.

ơ Pyrastrana, Hub.

Ameriana, $T r$.

Rosana, Frol., Steph.

Gerningiana, Haw.

o Congenerana, Hub.

Iporana, Haw., Steph.

Fuscuna, steph., var.

Piceana.

dै Piceana, Linn.

Xylosteuna, $H u b$.

\& Oporana, Linn.

Hermanniana, $w, v$.

Dissimilana, Bent.

Crategana.

\& Cratægana, $I I u b$.

o Roborana, IIub.

\& Branderiana, Steph.
TORTRICES, Linn.

ANELLA.

Anella, W. V.? Gn., Zinck.

Bipunctanus, Zell.

GALLERIA, Lat., Gn.

Cerelat.

Cerella, $G n$.

б Cereana, Linn.

\& Mellonella, Linn.

Xylosteana.

Xylosteana, Linn.?

Characterana, $H u b$.

Ubliquana, Steph., var.

Sorbiana.

Sorbiana, Hub.

Avellana, Haw., Steph.

Rosana.

Rosana, Linn., Haw.

Lævigana, $W \cdot V$.

ơ Avellana, Linn.

Acerana, $\boldsymbol{H u b}$.

O Oxycanthana, $H u b$.

Viburnana, Haw., var.

Branderiana, Haw., var.

Fuscana, Haw., var.

Nebulana, Steph., var.

Dumetana.

Dumetana, $T r$.

Cratægana, Ficy. (non $\boldsymbol{H u b}$.)

Transitana.

Transitana

Acerana, Haw., Sleph.

Cinnamomeana.

Cinnamomeana, $\boldsymbol{T r}$.

Croceana, Frol.

Heparana.

Heparana, $W . V$.

Carpiniana, $H u b$.

Ribeana.

Ribeana, $H u b$.

Cerasana, $\boldsymbol{H} u b$., var.

Grossulariana, Steph., var.

Corylana.

Corylana, $H u b$.

Textana, $H u b$.

UNIFASCIANA.

Unifasciana, Dup.

Croceana, Haw., Steph.

Semialbana.

Semialbana, $G n$.

Consimilana, $T r$. (non $H u b$.)

Modeeriana, Haw. (non Linn.)

Costana.

Costana, W. V.?

Gnomana, Hub. 131.

Betulana, Don.

Spectrana, $T r$.

Latiorana, Wilk., var.

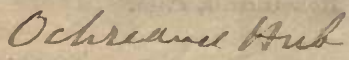

MELIPHORA, $G n$.

Alvedrielia.

Alveariella, $G n$.

Alvearia, $F a b$.

Cerea, Haw.

VibUrnana.

Viburnana, $W \cdot V$.

Unitana, $H u b .123$.

of Rhombana, $W$. $\boldsymbol{V}$.

Galiana, Bent., Steph.

ICTERANA.

Icterana, Frol.

Flavana, $H u b ., 258$.

Palleana, Wood, 816.

Viburnana, West.

Viridana.

Viridana, Linn.

Palleana, Steph. Mus. Cat., var.

Ministrana.

Ministrana, Linn.

Ferrugana, $H u b$.

Subfasciuna, Steph., var.

Adjunctana.

Adjunctana, $T r$.

Reticulana, Frol. (non $\mathrm{Hub}$.

Forsterana; Fab.? Steph.

Branderiana.

Branderiana, Linn.

Fuscana, $C l k$ :

Maurana, Hub., Steph.

Viduana, Dup, var.

Donzelana, Gn.

DICHELIA, $G n$.

Grotiana.

Grotiana, Fab.

Flavana, Hub., 133.

Ochreana, Steph., var.

AMPHYSA, Curt.

Gerningana.

Gerningana, $W . v$.

Pectinanu, Gn.

Walkerana.

Walkeri, Curt.

Prodromana, $H u b$ ?

ENECTRA, $G n$.

Prlleriana.

Pilleriana, $W$. $V$.

Luteolaua, $\boldsymbol{I} u \boldsymbol{b}$.

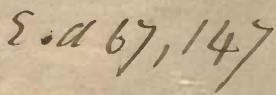




\section{LEPTOGRAMMA, Curt.}

LITERANA.

Literana, Linn.

Komanana, Fab., var.

Asperana, $W . V_{\text {., }}$ var.

Syuumana, $\boldsymbol{F} a b$.

Irrorana, Steph., var.

Tricoloranu, Steph., var.

Fulvomixtana, steph., var.

Treveriana.

Treveriana, $W . V . ?$

Scotana, Gn. MSS.

Scabrana.

Scabrana, Fab.

Parisiana, Gn.

Ulmana, Dup.

Boscana.

Boscana, Fab.

Cerasana, $H u b$, var.

\section{PERONEA, Curt.}

Faviluaceana.

Favillaceana, $\mathrm{Hub}$.

Reticulana, Haw., var.

Tristana, Haw., var.

Rufana.

Rufana, $W . V$.

Lucidana, $T r$.

Densana, Frey.

Albicostana, Steph., rar.

Bistriana, Haw., var.

Crassana, Dup.

Similana, Steph., var.

Atomella, Wood, var.

Lipsiana, Dbl. Cat., var.

\section{Mixtana.}

Mixtana, $H u b$.

Castaneana, Haw.

Schalderiana.

Schalleriaua, Linn.

Latifasciana, Huw., var.

Comparana, Hub., var.

Kufana, Haw. (non $W$. V.)

Costimaculann, Steph., vur.

Potentillana, Ćooke, var.?

\section{Caledoniana.}

Caledoniana, Bent.

\section{Permutana.}

Permutana, Dup.

Variegana.

Variegana, W. V.? Steph. A bildgaardana, Fub., Gn.

Asperana, Fab., var.

Nyctemerana, $H u b$.

Borana, Haw., var.

Cirrana, Curt., var.

Albana, West., var.

\section{Cristana.}

Cristana, W. V.

Cristana, Fab., var.

Ephippana, F'ub., var.

Lefeburiana, Dup.

Alboflainmana, C'urt, var.

Albipunctana, Steph., var.

Ruficostana, Curt., var.

Subvittata, Steph., var.

Capuzina, Johns., var.

Subcapucina, Desv., var.

Curtisana, Desv., var.

Insulana, Curt., var.
Curstana (continued).

Combustana, Dup., var.

Xunthovittana, Desv., rar.

Rossiana, Fab., vur.

Profauana, $F a b$., var.

Unicolorana, Desv.

Desfontianana, $F a b$, var.

Sericana, $H u b$., var.

Consimilana, Steph., var.

Lichenana, Curt., var.

Albovittana, Steph., var.

Fulvocristana, Steph., var.

Tolana, Desv., var.

Spadiceana, Haw., var.

Substriana, steph., var.

Brunneann, Steph., var.

Vittana, Sleph., var.

Chantaua, Curt., var.

Cristalana, Don., var.

Fulvovittana, Steph, var.

Subcristalana, Steph., var.

Sequana, Curt., var.

Striana, Haw., var.

Fulvostriana, Desv., var.

Assimilana, Steph, var.

Semiustana, Curt, var.

Bentleyana, Curt., var.

Hastiana.

Hastiana, Linn.

Scabrana, $W . V$.

Elevuna, Fab.

()bsoletana, Steph., var.

Scabrana, Hub., var.

Sparsana, $W$. $V$., var

Sponsána, Fab., var.

Aquilana, $H u b$, var.

Radiaun, Hub., var.

Strigana, Steph., var.

Divisana, Steph., var.

Rawostriana, Steph., var.

Centrovittana, Steph., var.

Mayrana, $H u b$., var.

Albistriana, Haw.,var.

Combustana, $H u b$., var.

Subcristana, Steph., var.

Autumnana, Steph., val.

Leucopheana, Bent., vur.

Sparsana, $T r$., var.

Coronana, Steph., var.

Eximiana, Haw., var.

Byringerana, Hub., var.

Maccana.

Maccana, Tr., Herr.-Sch.

Marmorana, Bent.

\section{UMBRANA.}

\section{Umbrana, $H u b$.}

Radiana, Dup.

Ferrugana.

Ferrugana, $W \cdot V$.

Proteana, $G n$.

Tripunctana, $\mathrm{Hub}$.

Gnomana, Haw., Steph.

Rufana, Hub., var.

Tripunctulana, Haw.

Bifidana, Haw., S'teph., vas.

Notana, Don., var.

Tristana.

Tristana, Hub.

Proteana, var. $G n$.

Trigonana, Steph, var.

Logiana, steple, var.

Boscana, Haw., Steph, var.

Plumbosana, Haw., var.

Aspersana.

Aspersana, $H u b$

Subtripunctulaua, Steph.
Sheruerdana.

Shepherdana, Steph.

TERAS, $T r$.

CaUdana.

Caudana, $\mathrm{Fab}$.

Effractana, $H u b, 175$

Emargana, Fab., var,

Caudana, Steph., var.

Excuvana, Haw., var.

(Icbracea, Steph., var.

Contaminana.

Contaminana, $\mathrm{Hub}$.

Ciliana, Hub., var.

Obscurana, Don.

Khoinbuma, Haw., var.

\section{DICTYOP'TERYX, Step.}

\section{ULIGINOSANA.}

Uliginosana, Bent., West.

Atrosignana, Herr.-Sch. 350

LeEFingiana.

Lœflingiana, Linn.

Plumbana, Hub., var.

Ectypana, Hub. 190, var.

Holmiana.

Holmiana, Linn.

BERgMAN NIANA.

Bergmanniana, Linn.

Forskaleana.

Forskaleana, Linn.

ARGYROTOZA, Steph.

Conwayana.

Conwayana, Fab.

Hoffmanseggana, $H u b$.

Subaurantiana, Sleph., var.

PTYCHOLOMA, Steph.

LECHEANA.

Lecheana, Linn.

PENTHINIDAE, Gn. DITULA, Steph.

Hartmanniana.

Hartmanniana, Linn.?

Limeana, $\boldsymbol{V} \cdot \boldsymbol{V}$.

Scriptana, $\boldsymbol{H} u \boldsymbol{b}$.

Semifasciana.

Semifusciana, Haw.

Acutana, $T r$.

Elutana, Dup.

PENTHINA, $T r$.

ANTITHESIA, Steph.

Picana.

I'icana, Frol.

Corticana, Hub., Steph.

Capræana, Dup. pl. 245, f. 4, b.

Betuletana.

Betulætana, Hau:

Capreana, Dup. pl. 245, f. 4 , a.

Leucomelaua, $G n$.

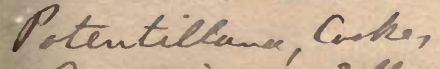

Comares auree 
Caprafana.

Capraana, $H u b$.

Pralongana.

Prelongana, Gn.

Betulætana, Herr.-Sch. 251.

Sororculana, Steph., var.

Pruniana.

Pruniana, $H u b$.

Pullana, Wood, var.

Ochroleucana.

Ochroleucana, $H u b$.

Tripunctata, Wood.

Cynosbana.

Cynosbatella, Linn.

Variegana, Hub., Gn.

Tripunctana, Haw., Steph.

Nubiferana, Haw., var.

Ochromei,ana.

()chromelana, $G n$.

Dimidiana, $\mathrm{Tr}$.

Weaverana, Dale, MSS.

SaUciana.

Suuciana, $H u \delta$.

Grevillana.

Grevillana, Curt. (an spec. dist.?)

Gentianana.

Gentianana, $H u b$. ?

Sellana.

Sellana, $H u . b$.

Pullana, Haw.? Steph.

Marginana.

ơ Marginana, Haw.

○ Oblongana, Haw.

Carbonana.

Carbonana, $D b l$.

Ustulana, Haw. (non Huk.)

ANTITHESIA, $G n$. PENTHINA, Stepl.

Salicana.

Salicana, $G n$.

Salicella, Linn.

SPILONOTID A, Gn. SPILONOTA, Curt.

Ocellana.

Ocellana, $W . V$.

Luscana, Fab.

Conjitana, Hub., Haw.

Pauperana.

Pauperana, Frey.

Paykulliana, Haw. (non Fab.)

Aceriana.

Aceriana, Mann.

Suciana, Haw., var.

Dealbana.

Dealbana, Frol.

Minorana, $T r$.

Sociana, Steph.

Incamana, Steph., var.

Paykulliana, Wood, 967, var.

Alnctana, Steph. Mus, Cat., var.

\section{Neglectana.}

Neglectana, Dup.

Sociana, Haw., var.

Simplana.

Simplana, Fisch.

A MANANA.

Amænana, Dup.

Suffusana.

Suffusana, Kollar.

Trimaculana, Haw.

Rosefelana.

Rosæcolana, Dbl.

Roborana.

Roborana, $W . V$.

Aquana, $H u b$.

Cynosbaua, $G n$.

PARDIA, $\boldsymbol{G} n$.

Tripunctana.

Tripunctana, $W . V$.

Ocellana, Hub.

Cynosbana, Haw.

\section{SERICORIDAE, Gn.} ASPIS, $T r$.

UDMANNIANA.

Udmanniana, Linn.

Rubiana, Scop.

Achatana, Hub. 49 .

SIDERIA, $G n$.

Achatana.

Achatana, $W . V$.

Marmorana, Hub., Steph.

\section{SERICORIS, $T_{r}, G_{n}$.}

\section{LATIFASCIANA.}

Latifasciana, Haw.

Grapholitana, $\boldsymbol{G} n$.

Aurofasciana, Hau.? Steph.

Veuustana, Hub.?

Dormoyana, Dup.?

Euphorbiana.

Euphorbiana, Zell.

Bifasciana.

Bifusciana, Haw., Steph.

Decrepitana, Fisch.

Litrorana.

Littoralis, Curt.

Venustana, Dougl. (non $H u b$, )

Morbidana, Gn. MSS.

Abscisana.

Abscisana, Gn, MSS .

Fuligana, Haw. (non Hub.)

Cespitana.

Cespitana, $H u b$.

Graminealia, Curt.

Conchana.

Conchana, $H u b$.

Undulana, $W$. V.? Steph.

HERBANa.

Herbana, $G n$.
LACUNANA.

Lacunana, $W . V$.

Micana, Haw. (nou Hub.)

Obsoletana, steph., var.

URTICANA.

Urticana, Hub.

DALEANA.

Daleana, $\mathrm{Dbl}$.

Alternana, Curt. (non W. V.)

Micana.

Micana, $H u b$.

Pinetana, Haw.

Haworthana, Steph.

MIXODIA, $G n$.

Schulziana.

Schulziana, $F a b$.

Piuetana, $H u b$.

Bentleyana, Don.

Zinckenana, $\boldsymbol{T} r$.

Palustrana.

Palustrana, Lienig.

Cespitana, Curt. (non $\boldsymbol{H u b . )}$

RAtzburghiana.

Ratzburghiana, Sux.

Pinetana, West. (non Hub.)

Tenerana, Dup. (non aliorum.)

Errana, $\boldsymbol{G} \boldsymbol{n}$.

BovchaRdana.

Bouchardana, Dbl.

Hawkerana.

Hawkerana, Stainton.

Consequana, Hut. 423 ?

ROXANA, Steph.

ARCCANa.

Arcuella, Linn.

EUCHROMIA, Steph.

Flammeana.

Flammeana, Frol.

Fulvipunctana, Haw.?

Purpurana.

Purpurana, Hau.

Rosuceana, Schlager

Arbutana.

Arbutella, Lipn

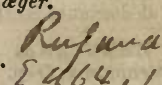

ORTHOTENIA, Steph.

AnTiquana.

Autiquana, $\mathrm{Hub}$.

Quadrimaculana, Haw.

Striana.

Striana, $W . \nabla$.

Rusticana, $H u b, 66$.

\& Fasciolana, Steph.

Enicetana.

Ericetana, Bent.

SCIAPHILIDAE, Gn. ERIOPSELA, $\boldsymbol{G} n$.

Fractifasciana.

Fractifasciana, Haw.

Cupliann, Fisch.

Caricana, $G n$.

Quecketana, Dale, Curt. 
Quanrana.

Quadrana, $H u b$.

PHTHEOCROA, Steph.

RUgosana.

Rukosana, $H u b$.

V-albana, Don.

\section{CNEPHASIA, Curt.}

Cinctana.

Cinctana, $W . V$.

Cretana, Fab.

Albidana, $H u b$.

LEPIDANA.

Lepidana, Curt.

Politana, Haw. (non $W . V$.)

Pulchellana, Haw., var.?

Musculana.

Musculana, $H u b$,

Trifasciana, Hau., Steph.

\section{SCIAPHILA, $\boldsymbol{T r}$.}

\section{CNEPHASIA, Curt.}

\section{Nubilana.}

Nubilana, $H u b$. ?

ơ Perfuscana, Haw.

\section{Perterana.}

Perterana, Gn., MSS.

Conspersana, Dougl. (non W. V.)

Cretaceana, Curt.

Decolorana, Steph. Mus. Cat.

Subjectana.

Subjectana, Gn., Steph. Mus. Cat. Logiana, Haw. (non Linn.)

Pasivana, Dul. Cat., var.?

Perplexana, Steph.Mus.Cat., var.

Virgaureana.

Virgaureana, $T r$.

Interjectana, Haw.?

Obsoletana, Steph., var.

Alternana.

Alternana, $W . V$

Asinana, Haw.

Chrysantheana, Dup.

Sinuana.

Sinuana, Steph.

Cinerana, Bent.

Stephensiana, $\boldsymbol{D} b l$.

HYBRIDANA.

Hybridana, $H u b$.

Reetifasciana, Haw.

Curvifasciana, Steph.

OCtomacUlana.

Octomaculana, Haw., Steph.

Penziana.

Penziana, Thunb., Steph.

Bellana, Curt.

Diurneana, $G n$.

Colquhounana.

Colquhounana, Stain.(præc, var.?)

SPHALEROPTERA, Gn.

\section{ICTERICANA.}

¿ Ictericana, Hav.

\& Longana, Haw.

Capillana, Gn.
CAPUA, Steph.

Ochraceana.

Ucbraceana, Steph.

Vulgana, Frol.

T'erreana, Dup.

CLEPSIS, $G n$.

RUsticana.

Rusticana, $\boldsymbol{T r}$.

GRAPHOLITHID AE, Gn.

BACTRA, Steph.

LaNCEOLANA.

Lanceolana, $H u b$.

Egenana, Haw., var.

Pauperana, Haw., var.

Egestana, Haw., var.

Plaganu, Haw., var.

Nigrovittana, Steph. Ms. Ct., var.

Furfurana.

Furfurana, Haw.

Fuscana, Churt. MSS.

Juscana, Gn., MSS.

\section{PHOXOPTERYX, $G n$.}

ANCHYLOPERA, Steph.

Siculana.

Siculana, $H u b$.

UNGUIQANA.

Unguicana, Linn. (ella.)

Fractifasciuna, Steph, (non Haw.)

UNCANA.

Uncana, $H u b$.

Geminana, Don.

Biarcuana.

Biarcuana, Steph. Cat.

Cuspidana, Steph., Ill.

Crenana, Dup. (non Hub.)

Subarcuana.

Subarcuana, Dougl.

Comptana.

Comptana, Frol.

Myrtillana.

Myrtillana, $\boldsymbol{T} r$.

Lyellana, $C u, t$.

Derasana, Steph.

LUNDANA.

Lundana, $F a b$.

Derasana.

Derasana, $\boldsymbol{H} u b$.

Uuculana, Haw., Steph.

Diminutana.

Diminutana, Haw.

Cuspidana, $\boldsymbol{T r}$.

Mitterbacheriana.

Mitterbacheriana, $W . V$.

Penkleriana, $T r$.

Subuncana, Haw.

Upupana.

Upupana, Tr. Supp.

Ustuluna, Her'r.-Sch.

Ramana.

Ramana, Linn.(ella)?

Haspana, Hub., Steph.
GRAPHOLITA, $\boldsymbol{T r}$.

Paykulliana.

Paykulliana, Fab.

Sesquilunana, Haw.

Triquetrana, $H u b .$, var.

Costana, Dup , var.

Nisana.

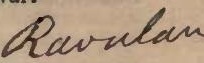

Nisana, Linn. (ella.)

Pavonaua, Don., var.

Silaceana, Hub.; vas.

Rhombifuscians, Haw., var.

Bœeberana, Huw., var.

Petrana, Hub, var.

Cusuidana, Haw.

Decorana. Hub., var.

Stictana, Haw., var.

Cinerana, Haw., var.?

Nigromaculana.

Nigronaculana, Haw.

Freyeraua, Fisch.

Albana, Haw., var.

Campoliliana.

Campoliliana, $W \cdot V$.

Subocellana, Don.

Decorana, Haw., var.

Asseclana, Steph.

Minutana.

Minutana, $\boldsymbol{H} u b$.

Trimaculana.

Trimaculana, $D o n$.

Mitterbachiana, Hau.

Ulmariana, Zell.

Excruciana, Steph. Mus. Ct., var.

Penkleriana.

Penkleriana, $W$. $\boldsymbol{V}$.

Retusana, Haw.

Obtusana.

Obtusana, Haw.

Distortana, $G n$.

Nevana.

Nævana, $H u b$.

Unipunctana, Haw.

Geminana.

Geminana, Steph.

Dorsivittana, Zell.?

PHI.EODES, $G n$.

Tetraquetrana.

Tetraquetrana, Haw,

Frutetana, Hub.

Angulana, Haw, var.

IMMUNDANA.

Inmundana, Fisch.

Triquetiana, Haw., var.

DEMARNIANA.

Demarniana, Fisch.

Nævana, Steph. (non Hub.)

Crenana.

Crenana, $H u b$.

Cognatana, Steph. Mus. Cat., var.

HYPERMECIA, $G n$.

Augustana.

Augustaua, $H u b$.

Cruciana, Linn.? Haw.

Excecana, Herr.-Sch.

Viminana, Gn., var. 
BATODES, $G \boldsymbol{n}$.

Angustioran..

Angustiorana, Haw.

Rotundana, Haw.

Dumeriliana, D $u p$.

PQE DISCA, Tr., Gn. PCECILOCHROMA, Ste.

Bilunana.

Bilunana, Haw.

Cretaceana, Frol.

Oppressana.

Oppressana, $T r$.

Corticana.

Corticana, $W . V$.

Iichenana, $T r$.

Communana, Steph.

Profundana.

Profundana, $W . V$.

Wellensiana, Hib., Steph.

Porphyrana, Hub., Haw., var.

Nebulana, Don., var.

Ethiopana, Haw., var.

Aseclana, Haw., var.

Sylvana, Haw., var.

Opthalmicana.

Opthalmicana, $H u b$.

Maculana, Fab.? Steph.

Occultava.

Recultana, Dougl.

Pinicolana, Zell. (Isis.)

Solandriana.

Solandriana, Linn.

Brunnichiana, Linn, var.

Parmatana, Hub. var.

Ratana, $H u b$, var.

Funalana, Steph., var.

Semifuscana.

Semifuscana, Haw.

Melaleucana, Dup.

Piceana, Haw., var.

Sordidana.

Sordidana, Hub. (præc. var.?)

Stabilaua, Steph. Mus. Cat.

\section{EPHIPPIPHORA, $G n$.} HALONOTA, Steph.

Bimaculana.

Bimaculana, Don.

Similana, Hub. 41.

Stræmiana, Haw.

Dissimilana, Fisch.

Cirsiana.

Cirsiana, Zell.

Mortuana, Gn. MSS.

\& Sticticana, var. $\beta$, Haw.

Scutulana.

Scutulana, $W . V$.

o. Pflugiana, Haw.

Novana, Gn., var.

\& Sticticaua, Haw., var. a.

Brennichiana.

Brunnichiana, $\boldsymbol{W}$. $V$.

Rusticana, Haw.

Quadrana, Steph., var.
Turbidana.

Turbidana, $T r$.

Foneana.

Fœneana, Linn. (ella.)

Scopoliana, W. $V$.

Nigricosita na.

Nigricostana, Huw.

Remyana, Koll.

Signatana.

Signatana, Dougl.

Padana, Lienig.

Trigeminana.

Trigeminana, Steph.

\& Argyrana, Steph.

Tetragonana.

Tetragonana, Steph.

Luctuosana, Dup., Gn.

Costipunctana.

Costipunctana, Haw.

Gallicolana, Heyden, Zell.

Ephippana.

Ephippana, $H u b$.

Populana, Fab.?

Obscurana.

Obscurana, Steph.?

OLINDIA, $G n$.

ULMANA.

Ulmana, $H u b$.

o Areolana, $\mathrm{Hub}$.

Hastiana, Steph. (non Linn.)

SEMASIA, $G n$.

Spiniana.

Spiniana, Fisch., Gn.

Ephippana, Steph.

JanTIIINANA.

Janthinana, Dup., Gn.

I.ediana, Steph.

Lathyrana, Steph. Mus. Cat.?

Rufillana.

Rufillana, Zell. in litt.

Lediana, Haw., var.

WeBerana.

Woberana, $W . V$.

Ornatana, $H u b$.

\section{COCCYX, $T r$.}

Cosmophorana.

Cosmophorana, $\boldsymbol{T}$.

Strobilana.

Strobilana, Linn. (ella.)

Splendidulana.

Splendidulana, $G n$.

Strobilaua, Haw.

Fraternana, Steph.

Argyrana.

Argyrana, $H u b$.

Atromargana, Haw.

o Strobilella, Steph.

TEDANA.

Tædana, Linn., Clk.

Finitimana, $G n . M S S$.
Pygmoana.

Pygmrana, Hub.? Gn.?

Subsequana, Haw.

Hyrciniana.

Hyrciniana. Uslar.

Comitana, Steph.

Distinctana.

Distinctana, Bent.

Piceana, West.

USTOM ACULANA.

Ustomaculana, Curt.

Pellicana, Gn.MSS.

Nanana.

Nanana, $\boldsymbol{T} r$.

Tenebrosana, Dougl.

Douglasana, Gn.MSS.

Vacciniana.

Vacciniana, Fisch.

Myrtillana, Chant. MSS.

HEUSIMENE, Steph.

Fimbriana.

Fimbriana, Steph.

PAMPLUSIA, $\boldsymbol{G} n$.

Monticolana.

Monticolana, Mann.

Subsequana, Steph.

Alticolana, Steph.

RETINIA, $G n$.

Buoliana.

Buoliaua, $W$. $V$.

Turionana, $\dot{H} a \dot{w}$.

Gemmana, $H u b$.

Pinicolana.

Pinicolana, $\mathrm{Dbl}$.

Turionella, Curt., Steph.

Turionana.

Turionana, Linn. (ella.)

Pinivorana.

Pinivorana, Zell.

Resinella, Steph.

Albionana,Gn., MSS.

Resinana.

Resinana, Linn. (ella.)

Duplana.

Duplaua, Hub.

Sylvestrana.

Sylvestrana, Curt.

CARPOCAPSA, $T r$.

SPLENdaNa.

Splendan a, Hub.

Grossina.

Grossana, Haw.

Fagilandana, Heyd.?

Pomonana.

Pomonana, Linn. (ella.)

OPADIA, $G n$.

Funebrana.

Funebrana, $T r$. 


\section{ENDOPISA, $G n$.}

Nebritana.

Nebritana, $T r$.

Nigricana, Fab.?

Prsana.

Pisana, $G n$.

Proximana, Haw.?

\section{STIGMONOTA, $G n$.}

\section{Lunulana.}

Lunulana, $W . V$.

Dorsana, Fab.

\section{INTERRUPTANA.}

Interruptana, Herr.-Schf.?

\section{Coniferana.}

Coniferana, Ratzb.

Fissana, Steph. Mus. Cat., var.

LEPLASTRIANA.

Leplastriana, Curt.

Gueriniana, Dup.?

HEEgERANA.

Heegerana, Dup.?

Perlepidana.

Perlepidana, Haw.

Schrankiana, Frol.

INTERNANA.

Internans, $G n$.

Composana.

Composana, $\mathrm{Fab}$.

\& Gundiana, Steph.

Dorsana, Steph.

WEIRANa.

Weirana, Dougl.

Renimitana.

Redimitana, $G n$.

Nitidana, Fab.? Haw.

Trauniaya.

Trauniaua, $W$. V.?

Floricolana, $H u b$.

\section{REgiana.}

Regiana, Zell.

Trauniana, Dbl. Cat.

Puncticostana.

Puncticostana, Steph.

Immaculana. $\boldsymbol{G} n$.

Germarana.

Germarana, $H u b$.

Atropurpurana, Haw.

Proximana, steph.

\section{C}

DICRORAMPHA, $G n$.

Politana.

Politana, $W . V$.

Simpliciana, Wood.

Atrinana.

Alpinana, $\boldsymbol{T r}$. (præc. var.?)

Jacquinana, Haw.

Strigana, Haw., var.
Sequana.

Sequana, $\boldsymbol{H} u b$.

Petiverana.

Petiverella, Linn.

Lunulana, Wood, var.

Sequana, Wood, var.

Stelliferana, Curt., var.

Ulicana.

Ulicana, $G n$.

Saturnana.

Saturnana, $G n$.

Plumbagana.

Plumbagana, $\boldsymbol{T} r$.

Strobilana, Dup.

Acuminatana.

Acuminatana, Zell.

Caliginosaua, Dbl. Cat.

Senectana.

Senectana, $G n$.

Simpliciana.

Simpliciana, Hau.

Caliginosana, $T r$.

Artemisiana, Bent.

Tanacetana.

Tanaceti, $S t n$.

Consortana.

Consortana, Steph.

\section{PYRODES, $G n$.}

Rhediana.

Rbediella, Linn.

Daldorfiana, $F a b$.

Aurana, Hub.

\section{CATOPTRIA, $G n$.}

Albersana.

Albersana, $H u b$.

Rheediella, Steph.

ULiceTANA.

Ulicetans, Haw.

Succedana, $T r$,

Lanceolana, Steph.

Asseclana, Steph., var.

JULIANA.

Juliana, Curt.

Nimbana, Fisch.

Microgrammana.

Microgrammana, $G n$.

H ypericana.

Hypericana, Hub.

Parvolana.
Parvulana, Wilk.

Wimmerana.

Wimmerana, $\boldsymbol{T r}$.

Maritimana, Dale.

Scopoliana.

Scopoliana, Haw

Carduaua, $G n$.

\section{HohentwarthiaNa.}

Hohenwarthiana, $W . V$.

Pupillans, Haw.

Cana, Haw., var.

Fulvana, Steph., var.

Coeimaculana.

Cecimaculana, $\mathrm{Hub}$.

Modestana.

Modestana, Herr.-Sch.?

Expallidana.

Expallidana, Haw.

Rufana, Steph.

Westwoodiana, Gn. MSS.

Citrana.

Citrana, $H u b$.

Pupitilana.

Pupillana, Linn.

Absinthiana, $H u$ ub.

Lacteana, Steph., var.
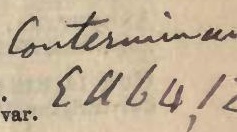

TRYCHERIS, $G n$.

Mediana.

Mediana, $W . \nabla$.

Aurana, Fab.

PYRALOIDAE, Gn. SIMAETHIS, Leach.

Vibrana.

Vibrana, Hub.

\section{CHOREUTES, $T r$.}

Scintilulana.

Scintilulana, $\boldsymbol{H u b}$.

Myllerana, Fab.? Haw

Punctosa, Hau., var.?

XYLOPODA, Lat.

Fabriciana.

Fabriciana, Linn.

Urticana, $W . \nabla$.

Dentana, Hub.

Parietariæ, Stn., var.?

Pariana.

Pariana, Linn.

Lutosa, Haw., var.

COCHYLIDAE, Gn. LOBESIA, $G n$.

Reciquana.

Reliquana, $H u b$.

Permixtana, Hub., Steph.

Servil laxa.

Servillana, Dup.

Paykulliana, West.

EUPCECILIA, Steph.

Nana.

Nana, Haw.

Carneana, Gin. 
Dubitana.

Dubitana, $\boldsymbol{H} u b$.

Atricapitana.

Atricapitana. Steph, Mus, Cat

Dubitana, Steph., Ill.

Maculosana.

Maculosana, Haw.

Sodalana.

Sodaliana, Haw.

Hybridellava.

Hybridella, $\boldsymbol{H} u b$.

Carduana, Zell.

Ambiguana.

Ambiguella, $\boldsymbol{H} u b$.

Angustana.

Angustana, $H u b .74$.

Curvistrigana.

Curvistrigana, Wilk.

Affinitana.

Affinitana, Dougl.

Vectisana, West., var.?

UDANA.

Uduna, $G n$.

Griseana, Haw.?

Notulara.

Notulana, Zell.

\section{Rupicolana.}

Rupicola, Curt.

Humidana, Fisch.

Flaviciliana.

Flaviciliana, Dbl.

Roseana.

Roseana, Haw.

Subrosfana.

Subroseana, Haw.

Ruficiliana.

Ruficiliana, Haw.

Ciliella, Hub.
Rubellana, Zell.

A NTHEMIDANA.

Anthemidana, Curt.

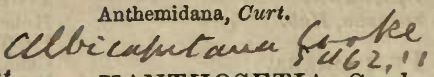

XANTHOSETIA, Steph.

\section{ZOEGANA.}

Zoegana, Linn:

Ferrugana, Haw., var.

Hamana.

Hamana, Linn.

Diversana, $H u \dot{b}$, var.

CHROSIS, $G n$.

Tesserana.

Tesserana, $W . V$.

Decimana, $H a w_{\text {, }}$ var.

Rutilana.

Rutilana, $\boldsymbol{H} u \bar{b}$.

Sanguinella, Haw.

Audouinana.

Audouinana, Dup.

Apicalis, West.

ARGYROLEPIA, Steph.

\section{BaUManNiana.}

Baumanniana, $W . \nabla$.

Hartmanniana, Clerck.

Dubrisana.

Dubrisana, Curt.

Virginana, Gn.

Marmorotana, Curt., var.

Luteolana, Steph., var.

Schreibersiana.

Schreibersiana, Frol.

Lediana, $H u b$.

Badiana.

Badiana, $\boldsymbol{H} u b$.

Smeathmanniana, Haw.

Rubigana, $T r$.

Cnicana.

Cnicana, $D b l$.

ENEANA.

Eneana, $H u b$.

Prapiciace Crut.

\section{TINEA.}

EPIGRAPHIDA, Gn. LEMNATOPHILA, $\boldsymbol{T r}$.

Phryganela.

Phryganella, Hub.

i Novembris, Haw.

SaIICELLA.

Salicella, $H u b$.

EXAPATE, Zell.

Gelatella.

Gelatella, Linn.
DIURNEA, Haw.

FAGELLA.

Fagella, $W . V$.

Dormoyella, Dup., var.

EPIGRAPHIA, Curt.

Avellanella.

Avellanella, $H u b$.

Steinkellneriella.

Steinkellneriella, $W . V$.
Mussenliana.

Mussehliana, $\boldsymbol{T r}$.

Maritimana.

Maritimana, $Q n$.

Cunices

COCHYLIS, $\boldsymbol{T r}$.

Dipoltana.

Dipoltella, $H u b$.

Margaritana, Haw,

Francillana.

Francillana, $\mathrm{Fab}$.

Flagellana, Dup.

Dilucidana.

Dilucidana, Steph. Mus. Cat.

Flagellana, Herr.-Sch. 95.

Smeathuanniana.

Smeathmanniana, $F a b$.

Fabriciana, $\boldsymbol{H u b}$.

Stramineana.

Straminea, Haw.

Tischerana, $\boldsymbol{T} r$.

Gigantana.

Gigantana, Gn.

Alternana, Steph. (non W. V.)

INOPIANA.

Inopiana, Haw:

Centrana, Herr.-Sch.

APHELIDAE, Gn. APHELIA, Curt.

Pratana.

Pratana, $H u b$.

Quadripunctana, Haw.

Cantiana, Curt., var.

\section{TORTRICODES, $G n$.}

Hyemana.

Hyemana, $H u b$.

万े Nubilea, Haw.

i Tortricella, Hub.
PSYCHIDAE, Bruand.

TALEPORIA, Zell.

Pubicornella.

Pubicornis, Haw. 2 Stn.

Pseudo-bombrcella.

Pseudo-bombycella, Och.

Tessellea, Haw.

PSYCHE, Bruand.

Villosella.

Villosella, Och, $B r$.

Nigricans, Curt., var. 
Opacella.

Opacella, Herr.-Sch., Br.

Fenella, Neum.

Calvella.

Calvella, Och., $\mathrm{Br}$.

Fusca, Haw.

Pullella.

Pullella, $B r$.

Pulla, Esp.

Radiella.

Radiella, Curt. (præc. var.?)

Reticella.

Reticella, Ncum., Br.

Roboricolella.

Roboricolella, $\mathrm{Br}$.

Nitidella, Steph.

Ce

SOLENOBIA, Zell.

Triguetrei.la.

Triquetrella, $\mathrm{Hub}$., $\mathrm{Br}$.

INCONSPICUELLA.

Inconspicuella, Stn, $\mathbf{B r}$.

Douglasii, Stn., var.? Cindmiectella Zell PSYCHOIDES, Bruand.

Verhuellella.

Verhuellella, Heyd.

TINEIDAE, Stn. DIPLODOMA, Zell.

MaRg inepunctella.

Marginepunctella, Steph.

Siderella, Zell.

XYSMATODOMA, Zell.

Melanella.

Melanella, Haw.

Atrella, Steph.

Argentimaculella.

Argentimaculella, Stn., Zell.

PHYGAS, $T r$.

OCHSENHEIMERIA, Zell.

Birdella.

Birdella, Curt.

Mediopectinellus, Haw.

Bisontella.

Bisontella, Lienig.

Taurella, Haw.?

Vaculella.

Vaculella, Fisch.

Chenopodiellu, Steph.?

SCARDIA, $\boldsymbol{T r}, \mathrm{G} n$

Chorarge Lla.

Chorargella, $W . \boldsymbol{V}$.

Boleti, Fab.

Mediella, Och.

Emortuella.

Emortuella, Zell.

Corticella, Curt,

Carpinetella.

Carpinetella, $G n$.

Parasitella, Hub.? Stn.
Picarelia.

Picarella, I,inn.

Rigaella, Sodoff.

Riganella, Herr.-Sch.

ArCuatella.

Arcuatella, Stn.

Picarella, Hub.

Granella.

Granella, Linn:

Cloacella.

Cloacella, Haw.

Ruricolella, Stn., var.?

Cochylidella.

Cochylidella, Stn.

Arcella.

Arcella, Fab.

Nivella, Fab.

Clematea, Haw.

TINEA, $S i n$.

IME LLA.

Imella, $H u b$.

Ferruginella.

Ferruginella, $H u b$.

Ustella, Haw.

Rusticella.

Rusticella. $H u b$.

Saturella, Haw.

Vestianella, Steph.

Lævigella, $W . V . ?$

MoNaChella.

Monachella, Hub.

Fulvimitrella.

Fulvimitrella, Sodoff.

Rupella, Haw., Steph.

Tapetzella.

Tapetzella, Linn.

Albipunctella.

Albipunctella, Haw.

Ca primulgella.

Caprimulgella, Herr.-Sch.

Misella.

Misella, Zell

Knockiella, Stn. Cat.

Pellionella.

Pellionella, Linn. 2 abiella

Fuscipuncteles.

Fuscipunctella, Haw.

Spretella, Zell.

Flatescentella.

Flavescentella, Haw. (præc. var. ?)

Pallescentella.

Pallescentella, $S t n$.

Ganomella.

Ganomella, $T r ., G n$.

Lapella, Stn.

Tripunctella, Don.

MerdelLa.

Merdella, Zell.

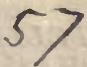

Biselliella:

Biselliella, Hemm.

Crinella, Tr.

Uestructor, Steph.
Simpliciella.

- Simpliciella, Herr.-8ch.

Nigripunctella.

Nigripunctella, Haw.

Parietariella, Herr.-Sch.

Semifulvella.

Semifulvella, Haw., Zell.

Bistrigella.

Bistrigella, Haw.

Abalienella, Zell.

Dilorella, Herr.-Sch.

Subamiranelua.

Subammanella, Stn., Zell.

Perochraceella.

Perochraceella, $\mathrm{Dbl}$.

Ochraceella, Tengs. (non Curt.)

LAMPRONIA, Zell., Stn.

Quadripunctella.

Quadripunctella, Fab.

Morosa, Zell.

LUZELLA.

Luzella, Hub.

Flavipunctella, Haw.

Pratatella.

Prælatella, $W . V$.

Mcestella, $\mathrm{Hub}$.

Luzella, $\boldsymbol{T r}$.

Flavinitrella, Dup.

Rubiella.

Rubiella, Bjerk.

Variella, Fab.

Corticella, Hawo.

Multipunctella, Dup.

INCURVARIA, Haw., St.

Masculella.

Masculella, $W . V$.

S Spuria, Haw.

Rutimitrella, West.

Zinckenella.

Zinckenii, Zell.

Pectinea, Fab.? Haw.

Tenuicornella.

Tenuicornis, Stn.

Oehrmanniella.

Oeblmanniella, $H u b$.

CA PITE LLA.

Capitella, Linn.

MICROPTERYX, Zell.

Calthella.

Calthella, Linn.

Sulcatella, Bent.

Aruncella.

Aruncella, Scop.

Seppella.

Seppella, Fab.

Eximiella, Zell.

o Calthella, Bent.

Mansuetella.

Mansuetella, Zell.

Allionella.

Allionella, $\mathrm{Fab}$.

Ammanella, $\mathrm{H} u b$. 
Thunberget.LA.

Thunbergella, Fab.

Rubrifasciella, Haw.

Hellwigella, Steph.

Purpurella.

Purpurella, Steph.

Rubro-aurella, Haw.?

Salopiella.

Salopiella, stn. (præc. var.?)

SEmipurpurella.

Semipurpurella, Stepk.

Solierella, Dup.

UNIMACULELLA.

Unimaculella, Zett.

Purpurella, var. b., Zell.

Sparmanella.

Sparmanella, Bosc.

Auropurpurella, Haw.

SubPurpurella.

Subpurpurella, Haw.

NEMOPHORA, Hub.

Swam Merdaue lla.

Swammerdamella, Linn.

Schwarziella.

Schw̋rziella, Zell.? Stn.

Nectella, Gu.

Carteri, Stn., var.?

Pilella.

Pilella, W. V.

Metaxflla.

Metaxella, $H u b$.

Robertella, Steph.?

ADELA, Lat.

Fibulella.

Fibulella, $W . T$.

Frischii, Haw.

Rufimitrella.

- Rufimitrella, Scop.? Stn.

Frischella, $H u b ., T r$.

Sulzella.

Sulzella, $W \cdot \boldsymbol{r}$.

Podaella, Don.

\section{Degeerella.}

Degeerella, Linn.

Viridella.

Viridella, Linn.

Cuprella:

Cuprella, Fab.

NEMOTOIS, Zell.

Scabiosella.

Scabiosella, Scop.

Cuprea, Haw.

Frischella, Curt.

\section{Cupriacella.}

Cupriacella, Hub.

Cypriacella, Dup.

SChIfFERMILLERELLA. Schiffermillerella, $W, \vec{V}$. Fasciella, Fab.
Minimella.

Minimella, Zell.

YPONOMEUTIDAE, St. SWAMMERDAMIA, $S t$.

Comptella.

Comptella, Hub.

A picella, Don? Stn.

Cesiflea.

Cæsiella, $\boldsymbol{H} u \bar{b}$

Heroldella, $H u b$. $\nabla$.

Griseocapitella.

Griseocapitella. Stn.

LUTARELIA.

Lutarea, Haw.

Pyrella.

Pyrella, Vill.

Cerasiella, Hub.

Cæsia, Haw. Imancers sat

SCYTHROPIA, Stn.

Cratagella.

Cratægella, Linn.

YPONOMEUTA, Lat.

ViginTIPUNCTELLA.

Vigintipunctatus, Retz., Zell. Sedella, $T r$.

Plumbella.

Plumbella, $W . \nabla$.

IRRORELLA.

Irrorella, $H u b$.

Padella.

Padella, Linn.

Cognatella.

Cognatella, Hub., Gr .

Evonymella, Scop.

Evonymelia.

Eronymella, Linn., G $n$.

Padi, Zell., Stn.

ANESYCHIA, Steph.

Funerella.

Funerella, Fab.

Decemautthlla.

Decemguttella, $\boldsymbol{H} u \boldsymbol{b}$.

CHALY BE, Dup.

Pyraustelila.

Pyrausta, Pallas.

PEPILLA, $\boldsymbol{G}$.

Curtisella.

Curtisella, Don.

Conobitella, Hub.

Rustica, Haw., var.

PLUTELIIDAE, Stn. EIDOPHASIA, Steph.

MessingIella.

Messingiella, Fisch.

Transversella, Stcph.
PLUTELLA, Schr.

Xylostella.

Xylostella, Linn. (F. S.)

Cruciferarum, Zell.

Porrectella.

Porrectella, Linn.

Hesperidis, Haw.

Annulatella.

Annulatella, Curt.

Speluncaricolella, Bruand.

Bicingulata, Herr..Sch.

Datella.

Dalella, Stn.

Vittella, Hub. 164 ?

Marmorosella, Wockc.

HYPOLEPIA, $\boldsymbol{G} \boldsymbol{n}$. CERosTOMA, $S t n$.

Sequelita.

Sequella, Linn.

Pusiella, Linn.? Gn.

VitTELLA.

Vittella, Linn.

Maurella, Steph., var.

Radiatella.

Radiatella, Don.

Fissella, Hub., Gn.

Variella, Hub.

Quinquepunctatus, Haw., var.

Lutosus, Haw., var.

Flaviciliatus, Haw., var.

Rufimitrellus, Steph.

Costella.

Costella, Fab.

Ochroleucus, Haw. var.

Ermineus, Haw. var.

Ustulatus, Haw.

YPSOLOPHA, $F u b$., $G n$.

SYLVELIA.

Sylvella, Linn.

Bifasciatus, Haw.

Alpella.

Alpella, $W . V$.

Persicella, Steph?

Lucella.

Lucella, $F a b$.

Antennella, $W . V$.

Mucronella, Hub. Haw.

HorridelLA.

Horridella, Tr., Gn.

Falcella, Steph.

Subfalcatella, Curt.

A SPE RELLA.

Asperella, Linn.

HARPIPTERYX, $T r . G n$.

Scabrella.

Seabrella, Linn.

Pterodactylellus, Haw.

Nemorelia.

Nemorella, Linn.

Hamella, Hub., Tr.

HARPELIA.

Harpella, $W$. $V$.

Dentella, Fab.

Xylostella, Stn. 
TINEA.

29

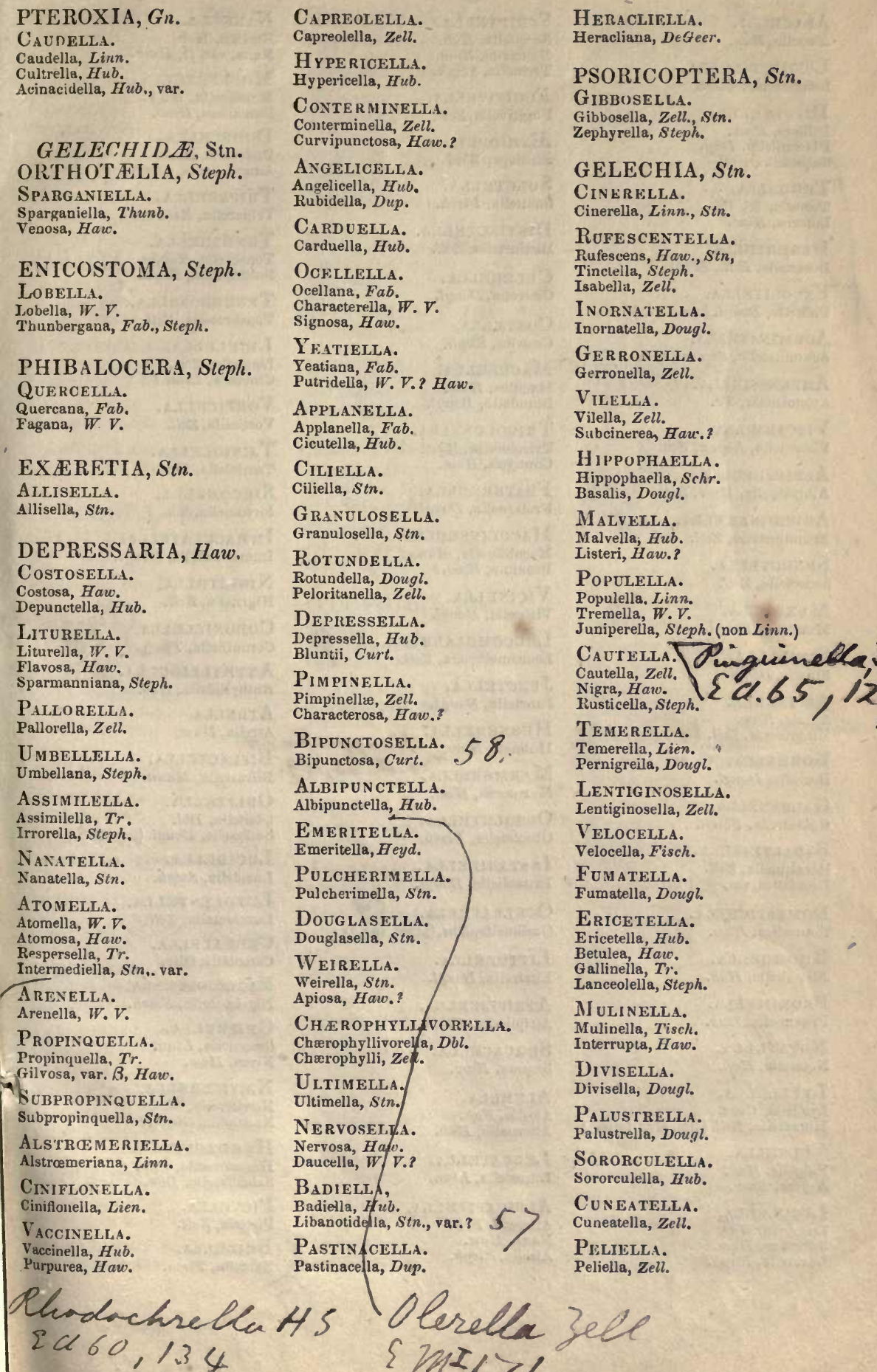




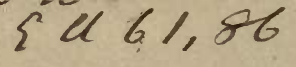

Alacelia.

Alacella, Dup.

LoNGfCoRNELLA.

Lougicornis, Curt.

DFFINELLA.

Diffinis, Haw.

Dissimilella, Dup.

Scabidella, Zell.

Terrella.

Terrella, $W . V$.

Lutarea, Haw.

Desertella.

Desertella, Edl., Stn.

Expolitella.

Politella, Dougl. (non Och.)

Acuminatella,

Acuminatella, sircom, stn. CuCla

ARtemisiella. $920 \mathrm{f} \& \alpha$

Artemisiella, $\mathbf{T}$ r. 70.97

VISCARIELLA.

Viscariella, Logan, Stn.

Albipalpella.

Albipalpella, Herr.-Sch. 58

Arundinetelua.

Arundinetella, Zell.

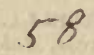

Senecteula.

Senectella, Zell.

Mundelta.

Mundella, Dougl.

Similella.

Similis, Dougl.

Affinella.

Affinis, Haw.

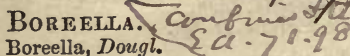

Gatbanelua.

Galbanella, Fisch.

Basaltinella.

Basaltinella, Zell.

Domestica, var. $\beta$, Haro.

Domesticella.

Domestica, Haw.

Rhombella.

Rhombella, $H u b$.

Proximeida.

Proximella, $\boldsymbol{H} u b$.

Notatella.

Notatella, Hub.

LYeILELLA.

Lyellella, Curt.

Humeralis, Zell. [non Hub. (ella.)]

Vulgella.

Vulgella, $H u b$.

Aspera, Haw.

LUCULELTA.

Luculella, $\boldsymbol{H} u b$.

Subrosea, Huw.

Luctuella, Steph.
Scriptella.

Scriptella, $\mathrm{Hub}$.

Blattariæ, Haw.

Tremella, Steph.

Fugitivelua.

Fugitivella, Zell.

Ethiopella.

Ethiops, West.

Solutella.

Solutella, Fisch.

Distinctella.

Distinctella, Zcll.

Celerella.

Celerella, Dougl.

Costella.

Costella, Steph.

Maculella.

Maculea, Haw.

Blandella, Dougl.

Tricolorella.

Tricolorella, Haw.

Contigua, Haw.

Fraternella.

Fraternella, Dougl.

MaCUliferella.

Maculiferella, Mann.

Proxima, Haw.?

VICINELLA.

Vicinella, Dougl.

Leucomelanella.
Leucomelanella, Zell.

Junctella.

Junctella, Dougl.

HubNerELla.

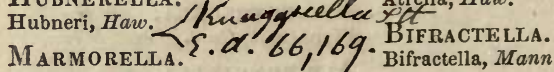

Marmorea, Hau.

Obsoletella. Obsoletella, Fisch.

INSTABILEILAA.

Instabilella, Dougl.

Ocellatella.

59

Ocellatella,, Stn, (præc. var. $\left.{ }^{\prime}\right)$

LitToRe Lla.

Littorella, Dougl.

Atriplicella. Atriplicella. Fisch

Sequacella. Sequax, Haro.

Ateella.

Aleella, $F a b$.

Alteruella, $\dot{H} u b$.

Leucatella.

Leucatella, Linn.

Albicapite LLA. Albiceps, Zell.

Nana, Haw.

Aleella, Steph.

Gemmeti.A.
NaNella.

Nanella, Hub.

Nana, var. $\beta$, Haw.

Mouffetella.

Mouffetella, $W . \nabla$.

Punctifera, Haw.

Dodecelta.

Dodecella, Linn.

Annulicornis, Steph.

TRIPARELla.

Triparella, Zcll.

Tenebrella.

Tenebrella, $H u b$.

Subcuprella, Steph.

Tenebrosklta.

Tenebrosella, Zell.

LIGULELLA.

Ligulella, Zell.

Cinctella, Linn.?

Vorticella.

Vorticella, Zell.

TENIOLELLA.

Tæniolella, $\boldsymbol{T r}$.

Sircomella.

Sircomella, Stn.

IMMACULATELLA.

Immaculatella, Dougl.

Nigrite Lla.

Nigritella, Zell.

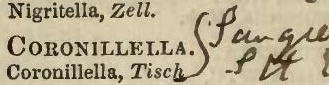

ANTHYLIIDE L LA. $63, !$
Anthyllidella, $H u b$.

Atrella.

Atrella, Haw.

Bifractella, Ma

Oblitella, $D b l$.

Suffusella, Dougl. (non Zell.)

IUCIDELLA.

Lucidella, Steph.

LUTULENTELLA.

Lutulentella, Zell.

Cerealelua.

Cerealella, Oliv.

Aigricostetia

Nigricostella, Fisch.

Gemmella, Linn.

Nivea, Haw.

NavifereluA.

Næviferella, Zell.

Knockella, Steph.?

Hermannella.

Hermannella, $\mathrm{Fab}$.

Schæfferella, Don.

Pictella.

Pictella, Zell.

Brizella.

Brizella, Tisch.

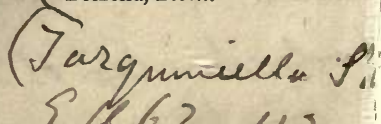


Subdecurtet.LA

Subdecurtella, stn.

Ericineila.

Ericinella, Zell.

Paupella.

Paupella, Zell.

INOPELLA.

Inopella, Zell.

Subocel le lla.

Subocellella, Steph.

K

PARASIA, Dup.

LAPPELLA.

Lappella, Linn.

Silacea, Haw.

Metzveriella.

Metzneriella, Dougl.

Silacea, var. $\beta$, Haw.

Carlinelua.

Carlinella, Dougl.

Neuropterella.

Neuropterella, Fisch.

CLEODORA, Sin.

Crtisella.

Cytisella, Cur't.

Fuscipennis, IVest.

Walkeriella, Dougl.

Striatella.

Striatella, $W . V$.

CHELARIA, Haw.

Conscriptella.

Conscriptella, $\mathrm{Hub}$.

Hubnerella, Don.

ANARSIA, Zell.

Spartiella.

Spartiella, schr.

Genistei.la.

Genistæ, Stn. (prøc, var.?)

MACROCHILA, Steph.

Fasciella.

Fasciella, $H u b$.

Marginella.

Marginella, $F^{\prime} a b$.

Clarella, $\boldsymbol{T} r$.

JuNiPERELLA.

Juniperella, Linn.

APLOTA, Steph.

Palpelia.

Palpella, Haw.

NOTHRIS, Stn.

VERBASCELLA.

Verbascella, $\boldsymbol{W} . \boldsymbol{V}$.

Durdhamella.

Durdhamella, Stn.
SOPHRONIA, Stn.

Parentrhesella.

Parenthesella, Linn.

Semicostella, Hub.

Homerella.

Humerella, Hub.

PLEUROTA, Stn.

Bicostella.

Bicostella, Linn.

HARPELLA, Schr.

Geoffrella.

Geoffrella, Linn.

Bracteella.

Bracteella, Linn.

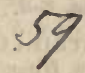

HYPERCALLIA, Steph.

Christiernella.

Christiernella, Linn.(ana.)

DASYCERA, $S t n$.

Sulphurella.

Sulphurella, $F a b$.

Orbonella, Hub.

Oliviella.

()liviella, Fab.

Emulella, Hub.

\section{ECOPHORA, Stn.}

Minute lla.

Minutella, Linn.

Oppositella, Fab.

Flavimaculecla.

Flavimaculella, Stn.

Minutella, Haw.

Trisignella.

Trisignella, Zell.

Tripuncta, Hau.

Stipella.

Stipella, Clerck.

Similella, Hub.? Stn.

Augustella.

Augustella, $H u b$.

Albimacciella.

Albimaculella, Haw. (præc. var.?)

Woodiella.

Woodiella, Curt.

Granderla.

Grandis, Desv.

Formosella.

Formosella, W. V.

LUNARELLA.

Lunaris, Haw.

Metznerella, $\boldsymbol{T r}$.

LAMBDELla.

Lambdella, Don.

Subaquile lla.

Subaquilea, Edl.

Tinctella.

Tinctella, $\mathbf{T r}$.
Subochreti.la.

Subochreella, $\mathbf{D b l}$.

Panzerella, Steph. (non W.V.)

Fusco-aurella.

Fusco-aurella, Haw.

Unitella, Stn. (non Hub.)

Arietella, Zcll.?

Flavifrontella.

Flavifrontella, $\boldsymbol{B} u b$.

Fuscescentella.

Fuscescens, Haw.

Pseudo-spretelld.

Pseudo-spretella, Stn.

CECOGENIA, Gn. MSS.

Kindermannielta.

Kindermanniella, Zell.

Quadripuncta, Haw. (non Fab.)

Bifasciella, Steph. (non $F a b$,)

Deauratella, Gn. MSS.

ENDROSIS, Stn.

Fenestrella.

Fenestrella, Scop.

Lactella, $W$. $V$.

Betulinella, Hub.

Sarcetia, Haw.

BUTALIS, $\boldsymbol{T r}$.

GrandipenNella

Grandipennis, Haw.

Fusco-feneella.

Fusco-ænea, Haw.

Senescentella.

Senescens, Stn.

Fusco-cuprella.

Fusco-cuprea, Haw.

Cica della.

Cicadella, Zell.

Varielia.

Variella, Steph.

Inspersella, Zell.

Chenopodiella.

Chenopodiella, Hub.

Cylindrea, Haw.?

Torquatella.

Torquatella, Lien.

INCONGRUELLA.

Incongruella, $S t n$.

PANCALIA, Stn.

LEUWENIIOEKELLA.

Leuwenhoekella, Linn.

Latreillella, Curt. ठ?

GLYPHIPTERYGID AE, St ACROLEPIA, Curt.

Perlepidella.

Perlepidella, Stn.

Ruficeps, Wocke. 
Granitel.la. Granitella, $T r$.

A utumitella. Autumnitella, Curt.

Pygmeana, Haw.

Heleniella, Zell.

Betcletla. C $6 / 8$ Betulella, Curt. MLabciclecla Curt

RÖSLERSTAMMIA, Stn.

Erxtebella.

Erxlebella, $\boldsymbol{F} a b$.

Fusco-cuprella, Haw.

Pronubella.

Pronubella, $W . \boldsymbol{V}$.

GLYPHIPTERYX, Slep.

Fuscoviride lita.

Fuscoviridella, Haw.

Albicostellu, Dup.

Thrasonella.

Thrasonella, Scop.

Fueslella, Fab.

Seppella, $H u b$.

Triguttella, Don.

Cladielta. Cladiella, Stn.

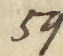

Haworthella.

Haworthana, Steph.

Zonella, Zett.

Ertitella.

Equitella, Scop.

SCHEN ICOLELLA.

Schœnicolella, Boyd.

Oculatella.

Oculatella, Zell.

Fischeriella.

Fischeriella, Zell.

ÆCHMIA, Sin.

Dentella.

Dentella, Stn.

PERITTIA, Sin.

Obscuripunctella.

Obscuripunctella, Stn.

Uleæ, Haw.?

TINAGMA, Dup.

Sericiella.

Sericiella, Hau,

Metallicella, Dup.

Stanneella.

Stanneella, Fisch.

Resplendella.

Resplendella, Dougl.

DOUGLASIA, $S \iota n$.

OcNerostomella.

Ocnerostomella, Stn.
ARGYRESTHID RE, St. ARGYRESTHIA, Str.

Erhippella.

Ephippella, $F a b$.

Pruniella, $H u b$.

Nitidella.

Nitidella, Fab.

Pruni, Haw.

Ossea, Haw., var.

Purpurascentella.

Purpurascentella, Stn.

Semtestacella.

Semitestacella, Curt.

Spiniella.

Spiniella, Zell.

Albistrie lla.

Albistria, Haw.

Conjugella.

Conjugella, Zell.

Semifuscella.

Semifusca, Haw.

Pruniella, Don.

Mendicella.

Mendica, Haw.

Tetrapodella, Zell.

Graucinella.

Glaucinella, $\boldsymbol{Z}$ ell.

Retinelica.

Retinella, Zell.

Abdominella.

Abdominalis, Zell.

Dilectella.

Dilectella, Zell.

Andereggiella.

Andereggiella, Fisch.

Curvella.

Curvella, Linn.

Cornella, $\mathrm{Fab}$.

Sorbiella.

Sorbiella, $T r$.

Pygmeella.

Pygmæella, $H u b$.

Semifasciella, Haw.

Gedartella.

Gœdartella, Linn.

Semiargentella, Don.

Lite RELIAA.

Literella, Haw. (præc, var.?)

Brockeella.

Brockeella, $H u b$.

Aurivittella, Haw., var.

Rajella, Linn.?

Aroevithinella.

Arceuthina, Zell.

Precocella.

Præcocella, Zell.

Aurulentella.

Aurulentella, Zell.

Decimella.

Decimella, Stn.

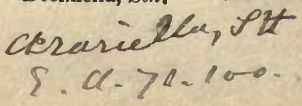

CEDESTIS, Stn.

Farinatella.

Farinatella, Zell.

Gysselinelua.

Gysselinella, Kuhl.

OCNEROSTOMA, Zell.

Pinarielia.

Pinariella, Zell.

ZELLERIA, Stn.

Hepariella.

Hepariella, Mann.

INSIGNIPE NNE LLA.

Insignipennella, Stn.

Fasciapennella.

Fasciapennella, Logan.

GRACILLARIDA, Stn. GRACILLARIA, Zell.

Swederella.

Swederella, Thunb.

Frankella, $H u b$.

Hilaripennella, Dup.

Thunbergella, Steph.

Stigmatella.

Stigmatella, $F a b$.

Upupæpennella, $H u b$.

Trigona, Haw.

()chracea, Haw.

Stramineella.

Stramineella, Stn.

Hemidacty lella.

Hemidactylella, $H u b$.

Falconipennella.

Falconipennella, $H u b$.

Semifascie lla.

Semifuscia, Haw.

Picipennella, $Z \in l l$.

Populetella.

Populetorum, Zell.

Elongella.

Elongella, Linn.

Signipennella, $H u b$.

Hemidactyla, Haw.

Tring ipennet, LA.

Tringipennella, Zell.

Roscipennis, Haw.?

SYRINGEL.LA.

Syringella, $F a b$.

Anastomosis, Haw.

Omissella.

Omissella, Dougl.

Phasianipennella.

Phasianipennella, $H u b$.

Auroguttella.

Auroguttella, Steph.

Lacertella, Zell.

Quadrupletla.

Quadruplella, Zell.

Eusp. Auroguttella, Steph.

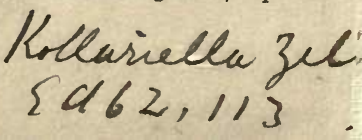


ONONIELLA.

Ononiella, Dup.

Ononidis, Zell.

IMPERIALELLA.

Imperialella, Mann.

\section{CORISCIUM, Zell.}

Brogniardelila.

Brogniardella, $F a b$.

Substriga, Haw.

Cuculipennella.

Cuculipennella, $\mathrm{Hub}$.

Cinerea, Haw.

Mandella, Dup.

Citrinella.

Citrinella, Fisch.

Sulphurella, Haw. (non Fab.)

Leucapennella, Steph. (non Hul)

ORNIX, Zell.

AVELLANACOLELIA.

Avellanella, Stn. (non $\boldsymbol{H} u b$.)

Devoniella.

Devoniella, Stn.

ANglicella.

Anglicella, Stn.

Betulavorella.

Betulævorella, $\mathbf{D b l}$.

Betulæ, Stn.

Scutolatella.

Scutulatella, Stn.

Torquilella.

Torquilella, Stn.

Scoticella.

Scoticella, Stn.

LOGANELLA.

Loganella, Stn.

Guttella.

Guttea, Haw.

COLEOPHORIDAE, St. GONIODOMA, Zell.

ATRIPLICIVORELLA.

Atriplicivorella, $\mathbf{D b l}$.

Auroguttella, Fisch. (non Steph.)

\section{COLEOPHORA, Zell.}

\section{Fabriciella.}

Fabriciella, Vill.

Spissicornis, Haw.

Mayrella, Zell.

Dea uratella.

Deauratella,Lien.

Trifolii, Steph.?

ALCYONIPENNF LLA.

Alcyonipennella, Koll.

Frischella.

Frischella, Linn.

Trifolii, Curt.

Paripfinella.

Paripennella, Fisch.

TINEÆ.

\section{Ea 61,89}

WOCKEELLA.

Wockeella, Zell.

Ochreella.

Ochrea, Hawo.

BinotapenNeLla.

Binotapennellu, Fisch.

LIXELLA.

Lixella, Zell.

Ornatea, Haw.

Ornatipennella, Steph.

Vibicella.

Vibicella, Hub.

Conspicuella.

Conspicuella, Mann.

PrRrhulipennelli.

Pyrrhulipennella, Tisch.

Albicostella.

Albicosta, Haw.

Volnerariella.

Vulnerariæ, Zell.

Anatipennella.

Anatipennella, $\mathrm{Hub}$.

Palliatella.

Palliatella, Zell.

IBIPENNELLA.
Ibipennella, Heyd.

Curructpennfella.

Currucipennella, Fisch.

Niveicostelia.

Niveicostella, Fisch.

Discordella.

Discordella, Z $Z$ ell.

GeNist ECOLELLA. 5
Genistæcolella; $D b l$.

Genista, Stn.

Saturatella.

Saturatella, Stn.

Onosmella.

Onosmella, Zell.

Struthionipennella, $\boldsymbol{T r}$.

INFLATELLA.

Inflatæ, Stn.

Therinelia.

Therinelle, Stn.

Troglodytella.

Troglodytella, Stn.

LiNEOLELLA.

Lineola, Haw.

Crocogrammos, Zell.

Murinipenvella.

Murinipennella, Fisch.

Souamosella.

Squamosella, $S t n$.

Cespitit iella.

Cæspititiella, Zell.

Tenast romella.

Tengstromella, $\mathrm{Dbl}$.

Annulatella, Teng. (non Curt.)

Salinella.

Salinella, Stn.

\section{Cacuminatelia.}

- Cacuminatella, Dbl.

Apicella, Stn. (non aliorum.)

Argentulella.

Argentula, Zell.

VIRGAUREFLLA. $5>$
Virgaureæ, $\mathrm{s} t n$.

HEMEROBIELLA.

Hemerobiella, Zell.

JunCicoleLLA.

Juncicolella, Stn.

LARICELIA.

Laricella, $\boldsymbol{H u b}$.

Albita rsella.

Albitarsella, Zell.

Nig RICELLA.

Nigricella, Steph.

Luscinæpennella. Bouche.

Coracipenuella, Zell.

Fuscedrelua.

Fuscedinella, Zell.

Orbitelica.

Orbitella, Zell.

GryphipenNelia.

Gryphipennella, Bouche.

Siccifoliella.

Siccifolia, Stn.

VITISELLA.

Vitisella, Greg. 57

Viminetella. Wheleune

Viminetella, Zell.

Outvaceelia. $\{\mathrm{Cl} 62 / 1\}$

Olivaceella, Stn.

Solitarielia.

Solitariella, Zell.

LUTIPENNELLA.

Lutipennella, Zell.

BaditPenNelia.

Badiipennella, Fisch.

LimosipenNella.

Limosipennella, Fisch.

Chalcogrammelica. 58

Chalcogrammella, Zell.

Chle

ELACHISTIDE, Stn.

BEDELLIA, Str.

Som N ULENTELLA.

Somnulentella, Zell.

STATHMOPODA, Zell.

\section{Pedella.}

Pedella, Linn.

Angustipennella, $H u b$.

COSMOPTERYX, Stn.

\section{DruRELLA.}

Drurella, Zell.

Zieglerella, II $u$ b.

Eximia, Hau: 
Liexigiella.

Lienigiella, Zell.

\section{BATRACHEDRA, Stn \\ Preangustella. \\ Præangustella, Haw. \\ Turdipennella, $\boldsymbol{T r}$. \\ Pinicolelia. \\ Pinicolella, Zell. \\ OINOPHILA, Steph. \\ V-FLAVELLA. \\ V-flava, Haw.}

CHAULIODUS, Tr.

\section{INSE CURELLA.}

Insecurella, $S t n$.

ILLIGERELLa.

Illigerella, Hub.

Cherophylletia.

Chærophyllella, Goe

Testaceella, $H u b$.

\section{LAVERNA, Curt.}

Paludicolella.

Paludicolella, $\mathrm{Dbl}$.

Propinquella, Stn. (non Tr.)

LACTEELla.

Lacteella, Steph.

Gibbiferella, Zell.

Miscella.

Miscella, $W . V$.

Staintoni, Sircom.

Contuhbate lla. 58
Conturbatella, $H u b$.

Raschiriella. 58 ,

Raschkiella, Fisch.

Stephensielita.

Stephensii, Str.

Epilobiella.

Epilobiella, Schr.

Fulvescens, Haw.

Nebulella, Steph.

Ochraceella.

Ochraceella, Curt.

Phragmitella.

Phragmitella, Bent.

Decorelta.

Decorella, Steph.

Subbistrigella.

Subbistrigella, Haw.

Sturnipennella, $\boldsymbol{T} \boldsymbol{r}$.

Hellerella.

Hellerella, Dup.

Atra, Haw.

Rhamnela.

Rhamniella, Zell.

CHRYSOCLISTA, Stn.

I.INNEELLA.

Linneella, $\boldsymbol{S} t \boldsymbol{}$.
Bimaóulelua.

Bimaculella, Haw.

Schrankella.

Schrankella, $H u b$.

Locupletella, Fisch.

Flavicapitella.

Flavicapitella, Haw.

HELIODINES, Stn.

Roesella.

Roesella, Linn.

ANYBIA, Stn.

IAANGIELLA.

Langiella, $\boldsymbol{H} u \boldsymbol{b}$.

ASYCHNA, Stn.

Profugeilat.

Profugella, Zell.

Modestella.

Modestella, Dup.

FuscocilrelLA.

Fuscociliella, $S t n$.

Eratella.

Eratella, Zell.

Terminelia.

Terminella, Dale.

CHRYSOCORIS, Curt.

Festaliella.

Festaliella, $H u b$.

Scissella, Curt.

ANTISPILA, H.-Sch.

Pfeifferella.

Pfeifferella, $F a b$.

Quadriguttella, Haw.

Treitschkieli.

Treitschkiella, Fisch.

STEPHENSIA, Sin.

Brunnichella.

Brunnichella, Linn.?

Magnificella, Zell.

ELACHISTA, $S t n$.

Gleichenelua.

Gleichenella, Fab.

Magnificella.

Magnificella, Tengs.

Apicipunctelita.

Apicipunctella, Str.

Albifrontella.

Albifrontella, $H u b$.

Holden Elta.

Holdenella, $E d l$.

Atricomella.

Atricomella, Stn.

LUTicomella.

Luticomella, Zell.

Fravicomelia.

Flavicomella, Stn.
Poella.

Ров, Dougl.

Kilmunella. Kilmunella, Stm.

Alpinelia.

Alpinella, Edl.

Cinereopunctella.

Cinereopunctella, Haw.

Trapezie lla.

Trapeziella, Sin.

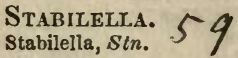

GregsonkLLA.

Gregsoni, Sin.

Nig Rella.

Nigrella, $H u b$.

SubNigrella.

Subnigrella, Dough

Perplexella.
Perplexella, Stn.

Humiliella.

Humilis, Zell.

Uccultella, Dougl.

Consortella.

Consortella, Stn:

Bedellella.

Bedellella, Sircom.

Nigrella, Dup.

Subonscuretra.

Subobscurella, $D b l$.

б' Obscurella, Stn. (non aliorum.)

\& Pulchella, Stn.

Zonariella.

Zonariella, Teng.

Gangabella.

Gangebella, Fisch.

\& Albinella, steph.

TeNIaTELLA.

Tæniatella, Stn.

Cingilella.

Cingilella, Fisch. 54

Obliquella.

Obliquella, Edl.

Megerlella.

Megerlella, Zell.

Adscitella.

Adscitella, stn.

\& Abruptella, Stn.

Cerussella.

Cerussella, Hub.

Rhynoosporflla.

Rhyncosporella, $\boldsymbol{S} t \boldsymbol{t}$.

Eleocharielta.

Eleochariella, Stn. (præc, var.?)

Biatomelia.

Biatomeila, Stn.

SERricornella.

Serricornis, Logan. 
Triatomenta.

Triatomea, Haw.

Despilella, $\boldsymbol{T} \boldsymbol{r}$.

Triseriatelia.

Triseriatella, $S t n$.

Coluiterla.

Collitella, Fisch.

Subocellea, Steph.?

Pollinarielda.

Pollinariella, Zell.

RUfociNerella.

Rufocinerea, Haw.

i Flos-lactis, Haw.

Subochreella.

Subochreella, $\mathrm{Dbl}$.

Ochreella, Stn. (non Haw.)

Cygnipennetita.

Cygnipennella, $\boldsymbol{H} u b$.

ơ Semialbella, Steph.

TISCHERIA, Zell.

Complanella.

Complanella, $\boldsymbol{H} u \boldsymbol{b}$.

Rufipennella, Steph.

Emyenta.

Emyella, Dup.

Marginea, Haw.

Angusticolella. 58

LITHOCOLLETID AE, St. LITHOCOLLETIS, Zell. ROBORELLA.

Roboris, Zell.

Hontella.

Hortella, Fab?

Saportella, Dup.

Amyotella.

Amyotellu, Dup.

LaNTANELLa.

Lantanella, Schr.

Triguttella.

Triguttella, $S t n$.

Quinqueguttelita.

Quinqueguttella, Stn.

Nigrescenter.LA.

Nigrescentella, Logan.

Irradiella.

Irradiella, Scott.

Bremiella.

Bremiella, Zell.

Lautella.

Lautella, Zell.

Vaccinielca. Vacciniella, Scott.

Cavelua.

Cavellu, Zell.

Pomifoliella.

Pomifoliella, Zell.
Conylelia.

Coryli, Nicelli.

Splvicolella.

Spinicolella, Zell.

Faginella.

Faginella, Mann.

ToRm Inella.

Torminella, Frey. 58

SAlicicolelta.

Salicicolella, Sircom.

Viminetella.

Viminetorum, Stn.

Carpinicolerla.

Carpinicolella, Stn.

ULMIFOLIE LLA.

Ulmifoliella, $\boldsymbol{H} u \boldsymbol{b}$.

Spinolella.

Spinolella, Dup.

Quercifoliella.

Quercifoliella, Fisch.

Messaniella.

Messaniella, Zell.

Corylifolie lla.

Corylifoliella, Haw.

Caledoniella.

Caledoniella, Stn.

Viminiella.

Viminiella, Sircom.

Scopariella.

Scopariella, Tisch.

Ulicicolella.

Ulicicolella, Vaughan.

Alnifol.Ie LLa.

Alnifoliella, $H u b$.

Heegeriella.

Heegeriella, Zell.

Cramerella.

Cramerella, Fab.

Tenelia.

Tenella, Zell.

ACERIFOLJELLA.

Acerifoliella, Zell.

Sylvella, Haw. (non Linn.)

EMBERIZÆPENNELLA.

Emberizæpennella, Bouch.

Frolichielda.

Frolichiella, Zell.

Dunningiella.

Dunningiella, $S t n$.

Nicellielda.

Nicellii, Zell.

Stettinella.

Stettinensis, Nicelli.

Klemannella.

Klemannella, $F a b$.

Schreberella.

Schreberella, Fab.
Tristrigella.

Tristrigella, Haw.

Trifasciella.

Trifasciella, \#law.

Scabiosecolella.

Scabiosæcolella, $\mathrm{Dbl}$.

Scabiosella, Dougl. (non Seop.)

Comparella.

Comparella, Fisch.

LYONETID AE, Stn.

LYONETIA, $H u b$.

Clerckella.

Clerckella, Linn.

Autumnella, Curt.

Nivella, Steph.

Padifoliella.

Padifoliella, Hub.8

PHYLLOCNISTIS, Zell.

Suffusella.

Suffusella, Zell.

Salig nella.

Salignella, Zell.

CEMIOSTOMA, Zell.

Spartifoliella.

Spartifoliella, $\boldsymbol{H} u b$.

LABURNELLA.

Laburnella, Heyden.

Scitella.

Scitella, Zell.

Clerckella, Steph.

WAILESELLA.

Wailesella, Stn.

LOTELLA.

Lotella, Stn.

OPOSTEGA, Zell.

Salaciella.

Salaciella, $T r$.

Auritella.

Auritella, $\mathrm{Hub}$.

Crepusculella

Pradute

BUCCULATRIX, $Z a l l, 13$

Aurimacolelia.

Aurimaculella, Stn.

Cidariella.

Cidariella, Tisch.

UlMELLA.

Ulmella, Mann.

Vetustella.

Vetustella, Mann.

Crategufoliella.

Cratægifoliella, Dup.

Cratæegi, Zell.

DEMARYELLA.

Demaryella, Dup,

Maritimelia.

Maritima, $S t n$. 


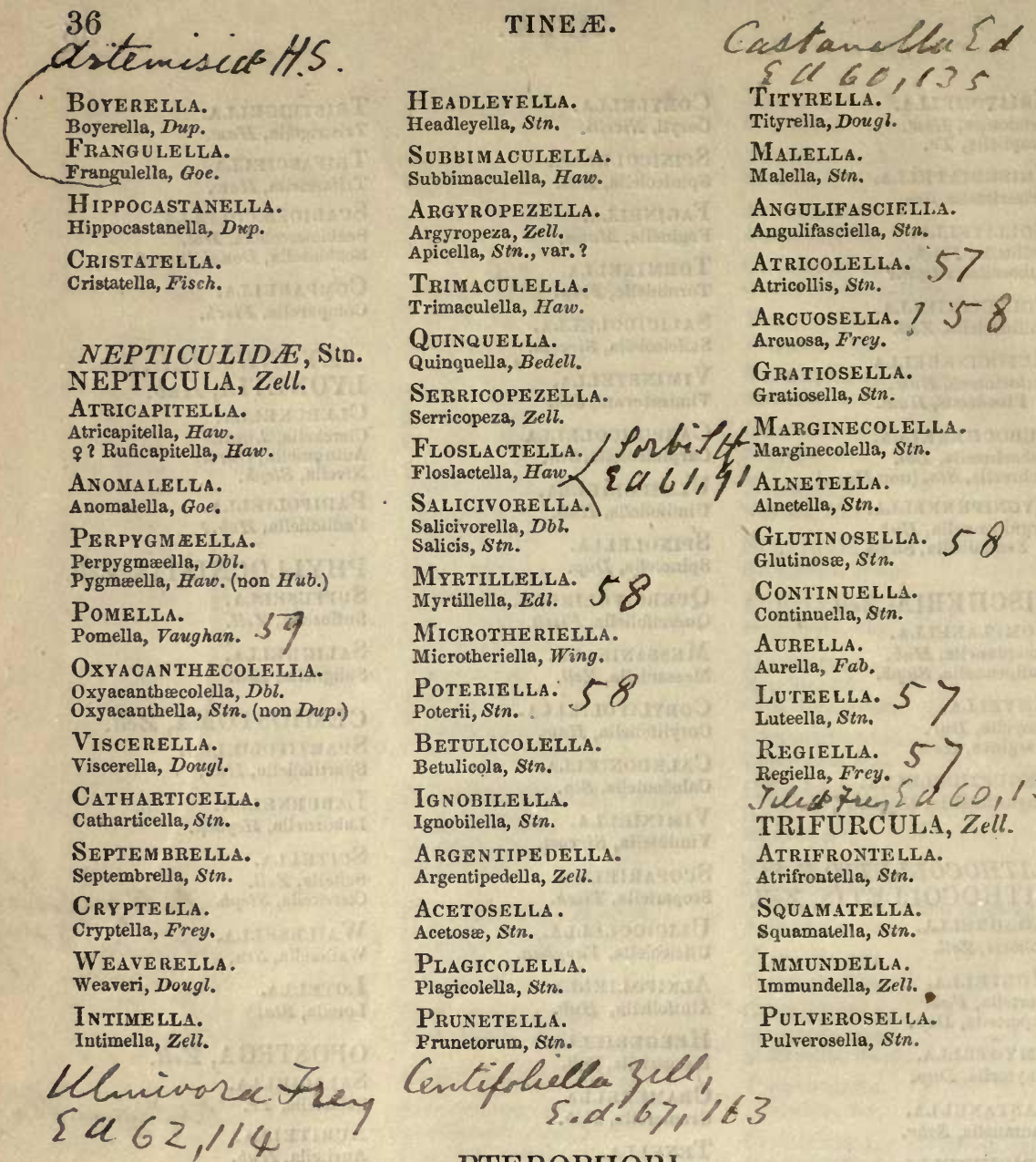

PTEROPHORI.

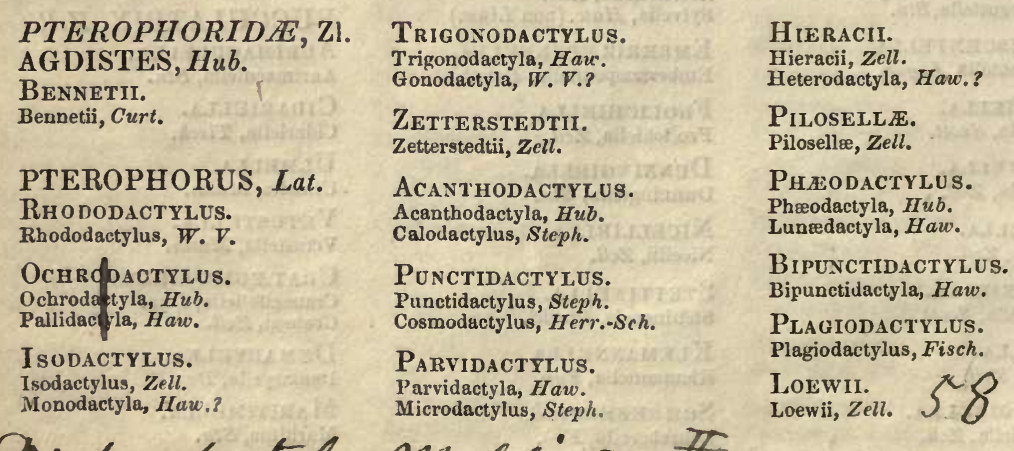

Dichrodactylus, mulalig $\left\{m^{\prime \prime} 33,165\right.$ 
FuscodactyuUs. Fuscodactylus, Vill.

Lithodactylus.

Lithodactyla, $\mathbf{T r}$.

Similidactylus, Dale.

Pterodactylus. Pterodactyla, Linn.

Lienigian US.

Lienigianus, $Z$ ell.

Tephradactylus.

Tephradactyla, $H u b$.

Osteodactylus.

Osteodactylus, Zell.
Microdactylus.

Microdactyla, $\boldsymbol{H} u b$.

Paludum.

Paludum, Zell.

BRACHYDAOTYLOB.

Brachidactyla, $T r$.

Galactodactyrus.

Galactodactylus, $H u b$.

SPilodactylus.

Spilodactylus, Curt.

Baliodatylus.

Baliodactylus, Zell.
Tetradactruus.

Tetradactyla, Linn.

Leucodactyla, $W . V$.

Pentadactylus.

Pentadactyla, Linn.

\section{$A L U C I T I D A B$.}

ALUCITA, Linn.

ORNEODES, Lat.

POLYDAOTYLA.

Polydactyla, Irub.

Hexadactyla, Linn.?

\section{ADDENDA ET CORRIGENDA.}

Page 3,

Genus SESIA,

For Altantiformis, read

ANDRENIFORMIS.

Andreniformis, Lasp.

Allantiformis, Newm.

\section{Genus NOLA, add}

Centonalis.

Centonalis, Hub.

\section{Page 12,}

Genus LUPERINA, Add after Testacea,

\section{Dumerili.}

Dumerili, Dup.

\section{After Cespitis, insert} CRYMODES, $G n$.

\section{Exulis.}

Exulis, Lef.

Gelata, Lef., var.

Groenlandica, Dup.

Borea, $B d v$., var.

Cervina, Germ., var.

Gelida, Gn., var.

Poli, Gn., var.

Assimilis, $D b i$., var.

\section{Page 15,}

Strike out Hadena assimilis, as synonymous with Crymodes exulis.

\section{Genus MICRA, After Ostrina, add}

Parva.

Parva, $H u b$.

Minuta, Dup.

$$
\text { Page 16, }
$$

Between the genera STILBIA and CATOCALA, insert

CATEPHIDA, $\mathrm{Gn}$.

CATEPHIA, Och.

A LCHYMista.

Alch ymista, $W . V$.

Between the genera RIVULA and HERMINIA, insert

SOPHRONIA, $G n$.

EMORTUALIS.

Emortualis, $W . V$.

\section{Page 18,}

Strike out Crambus lithargyrellus. The specimen under this name in the cabinet of the late J. F. Stephens being Crambus perlellus.

\section{Page 19,}

\section{Genus RHODOPHEA, insert}

Rubrotibiella.

Rubrotibiella, Mann.

\section{Page 27,}

Genus PSYCHE, add

\section{Salicolella.}

Salicolella, Bruand,

TABULELLA.

Tabulella, $G n$.

INTERME DIELLA.

Intermediella, $G n$. 


\section{REPUTED BRITISH SPECIES.}

The following species have either been placed in the British List without an authority, or specimens have only occurred under circumstances which rendered probable that they had been accidentally introduced.

\section{DIURNI.}

\author{
PAPILIO, Linn. \\ Podalirius. \\ Podalirius, Linn.

\section{PARNASSIUS, Lat.} \\ Apollo. \\ Apollo, Linn.

\section{COLIAS, $B d v$.} \\ Philodoce. \\ Philodoce, God. \\ Chrosotheme. \\ Chrysotheme, Esp.
}

\section{ARGYNNIS, Och.}

Nтове.

Niobe, Linn.

Dia.

Dia, Linn.

\section{SPHINX, Linn.}

\section{Carolina.}

Carolina, Linn.

Quinguemaculatus.

Quinquemaculatus, Haw.

Cingulata.

Cingulata, $\mathrm{Fab}$.

Druræi, Don.

\section{Pinastri.}

Pinastri, Linu.

DEILEPHILA, Och.

\section{Daucus.}

Daucus, $C r$.

Lineuta, Don.

\section{VANESSA, Och. \\ Huntera. \\ Huntera, Fab.}

EREBIA, $B d v$.

LIGEA.

Ligea, Linn.

SATYRUS, $B d v$.

M ERA.

Mæra, Linn.

Chortobius, $G n$.

Arcanius.

Arcanius, Likn.

Hero.

Hero, Linn.

\section{NOCTURNI.}

ZYGENA, Fab.

SCABIOSE.

Scabiosæ, $\boldsymbol{H} u \boldsymbol{b}$.

Meliloti.

Meliloti, Esp.

HipPocrepidis.

Hippocrepidis, Och.

CALLIMORPHA, Lat.

Hera.

Hera, Linn.

ARCTURUS, Curt.

Sparshalli.

Sparshalli, Curt.
POLYOMMATUS, $B d v$.

Virgaurez.

Virgaureæ, Linn.

Chryseis.

Chryseis, Fab.

LYCENA, $B d v$.

DoRYlas.

Dorylas, Hub.

Eros.

Eros, Och.

Icarius.

Icarius, Esp.

HESPERIA, $B d v$.

Vitellius.

Vitellius, $F a b$.

Bucephalus, Steph.

ORGYIA, Och.

V-NIGRA.

V-nigra, $F a b$.

BOMBYX, $B d v$.

Processionea.

Processionea, Linn.

Pytrocampa.

Pytiocampa, Fab.

EUTRICHA, Steph.

Pini.

Piui, Linn. 


\section{GEOMETRA.}

ACIDALIA, $T r$.

Herbariata.

Herbariata, Fab.

GNOPHOS, $T$ r.

\section{SERotinaria.}

Serotinaria, $W . V$.

Dilucidaria.

Dilucidaria, $W \cdot v$.

Pullata.

Pullata, $W \cdot V \cdot$ (non Steph.)

DASYDIA, $G n$.

Operaria.

Operaria, $H u b$.

Torvaria.

Torvaria, $K u b$.

DICRANURA, Lat.

ERMiNea.

Erminea, Esp.
PSODOS, $T r$.

Alpinata.

Alpinata, $W \cdot V$.

PELLONIA, Dup.

Vibicaria.

Vibicaria, Linn.

LYTHRIA, Hub.

Purpuraria.

Purpuraria, Linn.

ABRAXAS, Leach.

Pantaria.

Pantaria, Linn.
CLEOGENE, Dup.

LUTEaria.

Lutearia, Fab.

Tinctaria, $H u b$.

LARENTIA, $T r$.

Rupestrata.

Rupestrata, $W . V$.

OPORABIA, Steph.

Autumnata.

Autumnata, $B d v$.

EUBOLIA, Dup.

M enia RIa.

Mæniaria, $W . V$.

\section{PSEUDO-BOMBYCES.}

ClOSTERA, Hoff.

AnACHORETA.

Anachoreta, $W . V$.

Anastomosis.

Anastomosis, Linn.

\section{NOCTUA.}

ACRONYCTA, Och.

Cuspis.

Cuspis, $\boldsymbol{H} u b$.

Euphorbia.

Euphorbiæ, $W . \nabla$.

EUphrasiz.

Euphrasiæ, Bork.

SIMYRA, Och.

Nervosa.

Nervosa, $W . \nabla$.

XYLOPHASIA, Steph.

\section{LATERITIA.}

Lateritia, Esp.

HYDRILLA, $B d v$.

Palustris.

Palustris, Hub.

AGROTIS, Och.

Crassa.

Crassa, $H u b$.

ANNEXA.

Annexa, Och.

FENNICA.

Fennica, Tausc.

\section{XANTHIA, Och.}

Ocellaris.

Ocellaris, Bork.

DIANTHACIA, $B d v$.

CESIA.

Cæsia, $\boldsymbol{W}, \nabla$.

Albimacula.

Albimacula, Bork.

Сомтта.

Compta, Fab.

EcHII.

Echii, Bork.

VALERIA, Germ.

Oleagina.

Oleagina, $W . \nabla$.

MISELIA, Och.

Bimaculosa.

Bimaculosa, Linn.

C

CUCULlia, Och

Thapsiphaca.

Thapsiphaga, $T r$.

Luciruga.

Lucifuga, Esp.
NOTODONTA, Och.

Tonva.

Torva, Hub.

Querna.

Querna, $W . V$.
LaCtUCA.

Lactucæ, $E s p$.

Tanacetr.

Tanaceti, $F a b$.

Artemisie.

Artemisiæ, $F a b$.

CLEOPHANA, $B d v$.

Dejeanit.

Dejeanii, Dup.

CALOPHASIA, S'teph.

LiNARIE.

Linariæ, $W . V$.

CHARICLEA, Steph.

DelpirinII.

Delphinii, Linn.

HELIOTHIS, $O c h$.

Scutosa.

Scutosa, Fab.

ACONTIA, Och.

Aprica.

Aprica, $\boldsymbol{H} u b$.

Albo-ater, Haw.

Solaris.

Solaris, $W . V$. 
EUPHASIA, Steph.

Catena.

Catena, Sow.

PLUSIA, Och.

ILLUSTRIS.

Illustris, $F a b$.

Circumflexa.

Circumflexa, Linn, (non $H u b$.)

Flexuosa, Don.
TOXOCAMPA, $G n$.

LUSORIA.

Lusoria, $\operatorname{Linn}$.

LUDICRA.

Ludicra, $H u b$.

ANOPHIA, $G n$.

LEUCOMELAS.

Leucomelas, Linn.
CATOCALA, Och.

Elocata.

Elocata, Esp.

Pacta.

Pacta, Linn.

Conjuncta.

Conjuncta, $E s p$.

\section{DELTOIDES.}

HYPENA, Schr.

Obesalis.

Obesalis, $T r$.

\section{PYRALIDES.}

PYRALIS, Linn.

Pictalis.

Pictalis, Curt.
BOTYS, Lat.

Centro-strigalis.

Centro-strigalis, Steph.

Perpendiculalis, Dup.?

\section{CRAMBITES.}

CRAMBUS, Fab.

Tentaculellus.

Tentaculellus, $\mathrm{Hub}$.

Radiellus.

Radiellus, $H u b$.

'TORTRIX, Linn.

Croceana.

Crocerna, Hub.
INTERVIBRELLUS.

Intervibrellus, $G n$.

Tentaculellus, Wood.

LithaRgYRE LLUS.

Lithargyrellus, $H u b$.

\section{TORTRICES.}

Subocellana.

Subocellana, Steph.

Gromana.

Gnomana, Linn.

Biustulana.

Biustulana, Steph.
EUCHROMIUS, $G n$.

Ocellellus.

Ocellea, Haw.

Funiculella, $T r$.?

EPHESTIA.

Cerationella.

Cerationella, Fisch.

PERONEA, Curt.

LEACHEANA.

Leacheana, Curt.

SCIAPHILA, $\boldsymbol{T r}$.

WaH LBomiana.

Wahlbomiana, Linn.

\section{TINEA.}

ANESYCHIA, Sieph.

ECHIELLA.

Echiella, $W . V$.

Bipunctella, Fab.
LITH OSP ER M ELIA.

Lithospermella, $\mathrm{Hub}$.

Pusiella, Linn.? 
SUGGESTIONS FOR FORMING COLLECTIONS OF BIRDS' EGGS.-These 'Suggestions' are from the pen of Mr. Newton, than whom no one was ever better qualified for the task. The first object is to identify the egg, to be quite sure to what bird it belongs. Mr. NEwTox justly considers that an egg wrongly named is worse than no specimen at all. As soon as the egg is positively identified the next object is to authenticate it, by altaching such a mark as can neither be removed nor obliterated. Then follows a full and most minute description of the mode of blowing eggs, with figures of all the instruments necessary to be used; it is expressly explained that no hole sbould be visible, and it is also explained how to avoid this; on no account whatever should there be a hole at either end, and there should be but one hole. The difficulty of removing the contents frum eggs that have been sat on and nearly hatched is entirely overcome; ard the proper instruments to use are not only described but figured, and the requisite information is given where they may be obtained. Finally, we are shown how to strengthen the shell of delicate eggs before drilling the hole through which their contents are to be emptied.-Price $7 \mathrm{~d}$., post free.

\section{THE ZOOLOGIST LIST OF BRITISH BIRDS, compiled by the} late J. D. Salmon, F.L.S., from Mr. YARRELL's standard work on British Birds, comprising all the additions and corrections necessary up to the 1st of January, 1861. post free.

Price, printed on both sides, 3 d., post free; or on one side only for Labels, 5 d.,

THE ZOOLOGIST LIST of EUROPEAN BIRDS which do not occur in Great Britain.

Printed on one side only for Labels. Price $3 d$., post free.

AN ESSAY ON THE EMPLOYMENT OF PHYSIOLOGICAL CHARACTERS IN THE CLASSIFICATION OF ANIMALS. Being the substance of a Paper read before the Greenwich Natural History Club, December 6, 1856. By Eoward Newman, F.L.S., \&c., \&c.-Price 1s., post free.

A HISTORY OF BRITISH FERNS.-This work is illustrated by one hundred engravings, which are carefully executed by some of our first artists, from the author's own drawings on the wood, and consist of studiously accurate figures of every species and variety of fern found in Great Britain. Under eich species there is also a most minute description and ample list of localities, and full directions for cultivating. "It is just snch books as this which render Natural History so attractive to everybody who finds other pleasures in a country life besides hunting, coursing, fishing and shooting."-Gardener's Clironicle. "A valuable present to the lady botanist."-Gardener's Magazine. "No lady in the country ought to be without it." - Gardener's Gazette. Price 18s., post free.

BRITISH BUTTERFLIES.-Being a complete Natural History of these beautiful insects. By Edward NEWMaN, F.L.S., F Z.S., \&c., \&c. There is a life-size figure of every British Butterfly, drawn and engraved by the first Artists, regardless of expense, and a full description of each in the various stages of Caterpillar, Chrysalis and Butterfly; with an account of the food-plant, and full directions how to find, catch and preserve these Insects, and also a diagram figure, with explanations of the terms used.

This work has a portrait of the Author from a photograph by Messr8. Maull and Polyblank.-Price 7d., post free. 


\section{THE INSECT HUNTERS}

BY EDWARD NEWMAN, F.L.S., F.Z.S., LATE PRESIDENT OF THE ENTOMOLOGICAI SOCIETY.

I HAVE written this little book expressly for those who do not pretend to themselves experienced naturalists. I do not assume any knowledge on the part learner, but begin at the beginning, and attempt to educate the reader up to th of understanding my more complete and extended 'Familiar Introduction to the I of Insects;' indeed, I am not altogether without a hope that some will ci this inexpensive and unassuming book a sufficient Introduction to the Scif EDWard Newman.

"Unrivalled as a First-Book in Entomologr." - The late William F.R.S., one of the Authors of the celebrated 'Introduction to Entomology.'

"In the 'Insect Hunters,' by Mr. Newman, the author has brought togeth very pleasing way, a great deal of really good entomological matter bearing up habits, the economy and classification of insects. I can strongly recommen young persons who wish to obtain sound entomological information; and the tove in which the work is written is deserving of much commendation." - Adc William Wilson Saunders, Esq., to the Entomological Society of London, Anni Meeting, 1858.

"This is the first book from any author who understands the art of te Natural History pleasantly."-Report of the Northern Entomological Society.

"We know of no volume that contains so much information in so small a c on the habits and economy of insects." - Natural History Review, Edited by Haliday, Esq.

"Undoubtedly the best and most useful of Mr. Newman's entomological wo H. T. Stainton, Esq., Entomologist's Annual for 1858.

"There is a capital chapter on Metanorphosis, and the families in all the have their prominent characteristics concisely yet emphatically set forth.

For thE YOUNG who have not had their aitention drawn to Entomology we THIS AN ADMIRABLE BOOK, one that a parent might give to his child without a that the contents were not strictly true." - Entomologist's Weekly Intelligencer.

"The descriptions are accurate, and the author is well acquainted with the on which he writes." - Athenaum:

"The conception of this little work is novel, and its execution excellent likely to extend very widely the knowledge of Entomology. * * * We ha small compass and in the most captivating and easy form, all the information really essential to the study of Eutomolugy." - Literary Gazette.

"This is a little unassuming book, addressed by an experienced naturalis intelligent child, whom he leads by the hand through lanes, woods and mi showing her the living objects of his lessons, and explaining, in language ada childhood and youth, the habits and characters of each. We are spared all thost telligible technical explanations to which children will scarcely ever listen with pa and which they never remember." - The Friend.

"This work forms a complete introduction to the science of Entomology, writte elear and graceful language."-Kentish and Surrey Mercury.

Price 2s. 6d., post free.

Address, "Edward Newuan, 9, Devonshire Street, Bishopsgate, London, N.F 

Makers

Syracuse, $\mathbf{N}$. PAT. JaN 21, 1908

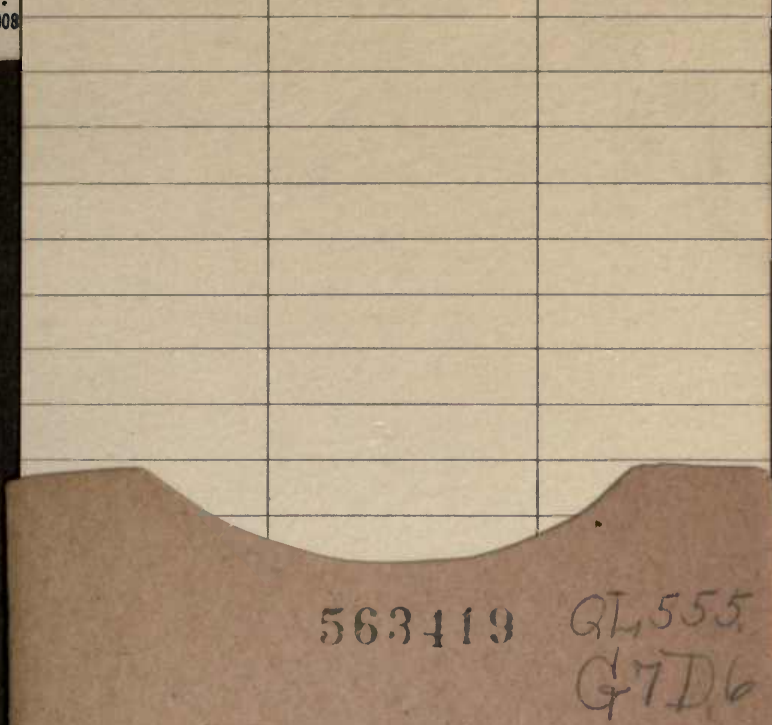

EIMLOSY EXERERY

G 
